University of Nebraska - Lincoln

DigitalCommons@University of Nebraska - Lincoln

January 1987

\title{
Electron transmission study of the temporary negative ion states of selected benzenoid and conjugated aromatic hydrocarbons
}

\author{
Paul Burrow \\ University of Nebraska - Lincoln, pburrow1@unl.edu \\ J.A. Michejda \\ Mason Laboratory, Yale University, New Haven, Connecticut \\ K.D. Jordan \\ Department of Chemistry, University of Pittsburgh, Pittsburgh, Pennsylvania
}

Follow this and additional works at: https://digitalcommons.unl.edu/physicsburrow

Part of the Physics Commons

Burrow, Paul; Michejda, J.A.; and Jordan, K.D., "Electron transmission study of the temporary negative ion states of selected benzenoid and conjugated aromatic hydrocarbons" (1987). Paul Burrow Publications. 14.

https://digitalcommons.unl.edu/physicsburrow/14

This Article is brought to you for free and open access by the Research Papers in Physics and Astronomy at DigitalCommons@University of Nebraska - Lincoln. It has been accepted for inclusion in Paul Burrow Publications by an authorized administrator of DigitalCommons@University of Nebraska - Lincoln. 


\title{
Electron transmission study of the temporary negative ion states of selected benzenoid and conjugated aromatic hydrocarbons
}

\author{
P. D. Burrow \\ Behlen Laboratory of Physics, University of Nebraska, Lincoln, Nebraska 68588-0111 \\ J. A. Michejda) \\ Mason Laboratory, Yale University, New Haven, Connecticut 06520 \\ K. D. Jordan \\ Department of Chemistry, University of Pittsburgh, Pittsburgh, Pennsylvania 15260
}

(Received 19 May 1986; accepted 23 September 1986)

\begin{abstract}
Electron transmission spectroscopy is utilized to determine the vertical electron affinities and to characterize the temporary anion states of a series of alternant hydrocarbons including benzene, naphthalene, anthracene, tetracene, styrene, and cis- and trans-stilbene. The vibrational structure present in the low lying resonances is interpreted in light of the charge distributions of the temporarily occupied orbitals. The energies of the anion states are compared with the predictions of PPP, PPP-CI, and HAM calculations, the pairing theorem and with the results from optical absorption measurements on the ground state anions in rigid glasses.
\end{abstract}

\section{INTRODUCTION}

Low energy electron transmission methods provide a sensitive means to study the formation of temporary negative ions in the gas phase and thus to probe the normally unoccupied orbitals of neutral molecules. ${ }^{1-3}$ Within the approximation of Koopmans' theorem ${ }^{4}$ the measured electron affinities (EA's) can frequently be associated with the energies of particular unfilled molecular orbitals.

Detection of negative ion formation via electron transmission spectroscopy (ETS) takes place by observation of the pronounced change in magnitude of the electron scattering cross section, or "resonance," at energies at which the impinging electron is temporarily captured into a normally unfilled orbital. ETS is thus restricted to those anion states which lie in the continuum, that is, whose energies lie above the ground state of the neutral molecule. For a great many of the smaller hydrocarbons such as ethylene, acetylene, formaldehyde, and benzene, vertical attachment into the lowest unoccupied orbital gives rise to anions which are unstable in the gas phase with lifetimes of $10^{-14} \mathrm{~s}$ or less. ${ }^{2}$ The range of anion lifetimes generally encountered extends from times comparable to vibrational periods to substantially shorter values. The existence and visibility of vibrational structure in the ET spectra are closely tied to the energies, spatial characteristics, and symmetries of the unfilled orbitals.

In several short publications, we have reported the results of ET studies of temporary anion states in ethylene, ${ }^{5}$ substituted benzenes, ${ }^{6(a)}$ butadiene, ${ }^{5}$ cis- and trans-1,3,5hexatriene, ${ }^{7}$ and styrene. ${ }^{6(b)}$ In the present work we extend these studies to naphthalene, anthracene, tetracene, and cisand trans-stilbene. We also present improved data for benzene and styrene.

\footnotetext{
") Present address: Bell Laboratories, Murray Hill, NJ 07974.
}

The molecules examined in this work are all alternant $\pi$ electron systems; that is, if one identifies alternating positions on the skeleton with an asterisk, no two adjacent positions are both "starred" or "unstarred." have been the subject of numerous experimental and theoretical studies. Many of their unique characteristics have been interpreted in terms of the pairing theorem ${ }^{8-10}$ which states that the quantity (IP + EA), where IP is the ionization potential, is a constant for all conjugate $\left(\pi, \pi^{*}\right)$ pairs in the molecule. This implies that the excitation spectrum of the ground state anion should be identical to that of the cation. It is important to note that although the pairing theorem is rigorous for the Hückel and Pariser-Parr-Pople (PPP) model Hamiltonians, it is not necessarily a property of the all-electron Hamiltonian. Nevertheless there is remarkable agreement between the condensed phase absorption spectra of the anions and cations of alternant hydrocarbons. The spectra of the cations in the gas phase are also in good agreement with those in condensed phases. However because of lack of data, it has not been possible to compare the gas phase anion spectra with the solution results or with the gas phase cation spectra. With ET studies, it is now possible to carry out such comparisons. ${ }^{11}$

In the following section we review the electron transmission technique and discuss the interpretation of ET data. In Sec. III we describe our results for each of the molecules. In the final section of the paper, we draw our conclusions.

\section{EXPERIMENTAL TECHNIQUE AND INTERPRETATION OF ET DATA}

The electron transmission technique devised by Sanche and Schulz ${ }^{3}$ has been described in detail in the literature. In this section we review the essential features and call attention to some aspects which should be emphasized. 
The transmission apparatus consists of a trochoidal electron monochromator ${ }^{12}$ which injects a magnetically collimated beam of electrons with current $I_{0}$ into a chamber of path length $L$ containing gas at density $N$. A portion of the scattered electrons is rejected electrostatically at a retarding plate following the collision chamber by discriminating against those electrons with reduced components of axial velocity. Under conditions in which all scattered electrons are rejected, the current transmitted to the collector is given by $I_{0} \exp (-N Q L)$, where $Q$ is the total scattering cross section.

The visibility of small variations in the cross section is increased by means of a modification introduced by Sanche and Schulz ${ }^{3}$ whereby the energy of the impinging electrons is modulated by applying a small ac voltage to a cylinder contained within the collision chamber. By detecting the resulting ac component of the transmitted current synchronously, a signal proportional to the derivative with respect to energy of the transmitted current is produced. The rapid variations in the cross section, which are due to temporary anion formation, are thereby greatly enhanced with respect to the broad, slowly varying features which result from nonresonant potential scattering.

In the usual mode of operation with a potential barrier imposed at the retarding electrode, the transmitted current is inversely related to a partial cross section for electrons scattered into a large solid angle. If the potential barrier is removed, rejection of a portion of the scattered electrons may still take place by other mechanisms. In particular, electrons elastically scattered into a small solid angle around $180^{\circ}$ can re-enter the crossed field region of the monochromator where they are dispersed and lost. Consequently the measured transmission signal is related to the differential cross section integrated over an energy-dependent solid angle centered on the back direction. ${ }^{13}$ This mode of operation is useful because the signal derived from the differential elastic cross section near $180^{\circ}$ often displays the vibrational structure in the resonances more distinctly for reasons described elsewhere. ${ }^{13}$

Calibration of the electron energy scale in this work has been carried out by admixture of the compound of interest with argon and referencing to the lowest Feshbach resonance in argon, located by Brunt et al.$^{14}$ at $11.098 \pm 0.010$ $\mathrm{eV}$. We have taken the midpoint between the two major extrema in the derivative to lie at $11.098 \mathrm{eV}$. This is not strictly correct, but because of the very narrow natural width of the resonance, $2.5 \pm 0.5 \mathrm{meV}$, and the nature of the resonance profile ${ }^{14}$ this assumption leads to an error well within our quoted limits of $\pm 0.03 \mathrm{eV}$.

In the present work we have employed two spectrometers to encompass the range of temperatures required for the subject molecules. The first machine was constructed largely as described by Sanche and Schulz, ${ }^{3}$ although the entrance and exit apertures of the collision chamber were enlarged to improve the operating characteristics when high rejection of the scattered electrons is used. The second machine contains an oven inside the vacuum envelope which is connected directly to the collision chamber. ${ }^{15}$ Each spectrometer employs a trochoidal monochromator ${ }^{12}$ with energy resolution of typically $30 \mathrm{meV}$ full-width at halfmaximum.

Although the sharp structure in an electron transmission spectrum may be located accurately, association of the energies of particular features with the electron affinities of the neutral molecule is considerably less certain. It is appropriate to mention the most important of the assumptions which go into these determinations before proceeding to a discussion of the results.

In cases in which sharp structure due to electron attachment into vibrational levels of the anion is observed, the vertical attachment energy may be reasonable assigned to the midpoint of the largest of the vibrational structures. In the limit that the lifetime is long compared to the vibrational period and the structure is instrumentally resolvable, the relative sizes of these features approach those expected from Franck-Condon considerations. ${ }^{16}$ For the intermediate lifetimes typically encountered in the shape resonances, however, the resonance features are usually broader than the instrumental resolution and overlap each other. In the absence of a clear-cut largest member, the vertical attachment energy as determined by the location of the midpoint between the extrema in the derivative could be in error by approximately the spacing between levels.

For most shape ${ }^{1}$ resonances encountered above impact energies of $2 \mathrm{eV}$, there is no vibrational structure appearing in the ET spectrum. We, as well as others, have customarily assigned the vertical attachment energy to the energy of the midpoint between the sometimes widely separated extrema. In these cases the breadth of the structure arises both from the finite lifetime $e^{1,15}$ of the anion and from factors related to the shape of the anionic potential surface. Both of these aspects however may cause the profile in the cross section to be skewed.

Finally, we return briefly to the case in which vibrational structure appears in the spectra and comment on the determination of the adiabatic electron affinity, that is, the energy from the zero point level of the neutral to that of the anion. As in other types of spectroscopy, such a procedure can be used unambiguously only when one is certain the lowest vibrational level of the anion has been observed. In earlier work we implicitly assumed that the distortion following electron capture was small, and that the first vibrational feature corresponded to the zero point level. However, this assumption is not generally valid and requires some clarification. The attachment process takes place into the portion of the anion potential energy surface which is accessible, in a Franck-Condon sense, from the ground state of the neutral molecule. If the potential energy surface of the anion is greatly stabilized by distortion along one or more coordinates, attachment at the adiabatic energy will not be possible. A prototypical example is $\mathrm{CO}_{2}$ in which a vibrational progression in the symmetric stretch mode of the anion starts at $3.1 \mathrm{eV},{ }^{3(\mathrm{c}), 17}$ whereas the adiabatic attachment into the bent molecule occurs at $0.6 \pm 0.2 \mathrm{eV} .{ }^{18}$ Such effects may also appear in small nonrigid hydrocarbons such as ethylene and butadiene. Larger molecules in which the $\pi^{*}$ orbitals are delocalized over many carbon atoms should undergo much less distortion. 


\section{RESULTS}

\section{A. Benzene, naphthalene, anthracene, and tetracene}

\section{Benzene}

The negative ion states of benzene have been studied by a number of investigators using electron transmission techniques, ${ }^{3(c), 3(d), 6(a), 19-23}$ and two prominent resonances are well known near 1.1 and $4.8 \mathrm{eV}$. Figure 1 illustrates the vibrational structure in the lower resonance using two different retarding voltages. In the curve labeled "high rejection," the potential on the retarding plate is only a few tenths of a volt more positive than that of the filament to achieve rejection of most of the scattered electrons. In the curve labeled "low rejection," the retarding barrier is considerably reduced by biasing the retarding electrode $\sim 3 \mathrm{~V}$ more positive than the filament. In this mode of operation, as discussed elsewhere, ${ }^{13}$ the primary rejection mechanism at low impact energies arises from those electrons scattered elastically into a cone centered at $180^{\circ}$. The visibility of the vibrational structure changes somewhat with rejection. The low rejection spectrum displays a small, $\approx 5 \mathrm{meV}$, shift of the apparent vertical attachment energy as well as some differences in vibrational spacing. This is likely due to the difference in the resonance profile in the differential and total cross sections.

To summarize, the structure appearing between electron impact energies from 1 to $2 \mathrm{eV}$ corresponds to the capture of an incident electron into the doubly degenerate $e_{2 u}\left(\pi^{*}\right)$ orbital, yielding the $X^{2} E_{2 u}$ ground state of the benzene anion. The angular distribution measurements of Wong and $S c h u l z^{24}$ demonstrate that near $1 \mathrm{eV}$ the scattered electrons display predominantly $f$-wave character, consistent with the leading partial wave in an expansion of the charge distribution of the $e_{2 u}\left(\pi^{*}\right)$ orbital. ${ }^{25}$ Further support for this assignment is provided by the observation that monosubstituted benzenes such as phenol and aniline display two

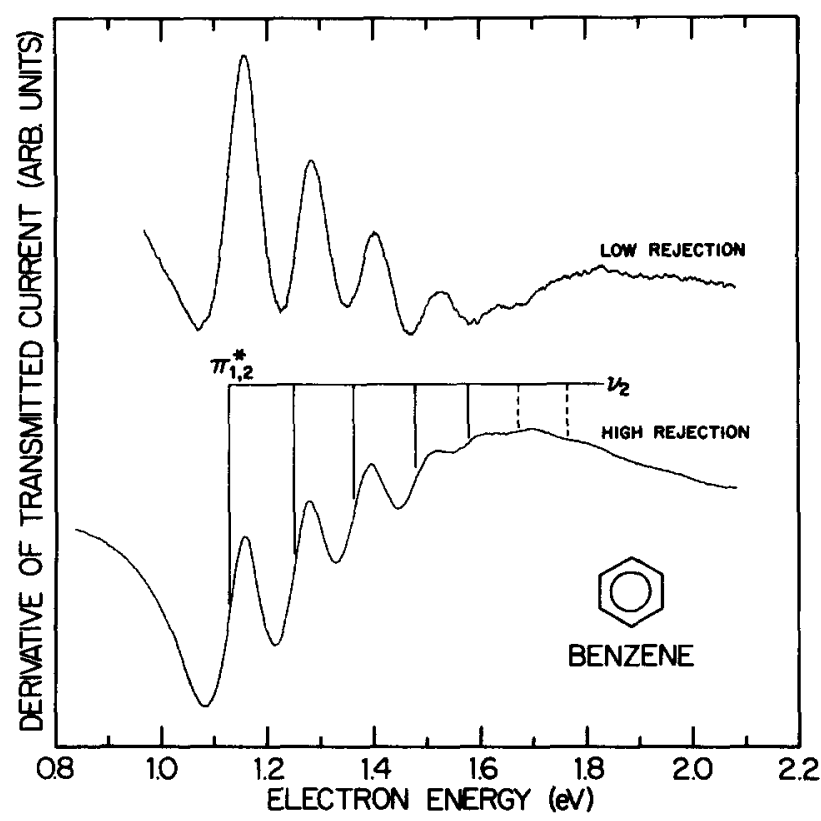

FIG. 1. Electron transmission spectrum of benzene near the ${ }^{2} E_{2 u}$ resonance. negative ion states at low energy, indicating that the structure in benzene originates from a doubly degenerate state. This splitting was first observed ${ }^{26}$ using the trapped electron method $^{27}$ and more recently by ETS. ${ }^{3(d), 20}$ The theoretical results given in Table I also provide additional support for this assignment.

The lifetime of the ground state anion is sufficiently long that a progression of vibrational levels attributed to the totally symmetric breathing mode, $v_{2}\left(a_{1 g}\right)$, is observed in the transmission spectrum. ${ }^{3(\mathrm{c})}$ The spacing between the first two levels of the anion as observed in the higher rejection spectrum is $123 \mathrm{meV}$, the same as that reported by Sanche and Schulz. ${ }^{3(\mathrm{c})}$ The spacing is also the same as that observed in the ground state of neutral benzen $e^{28}$ and close to that in the ground state cation ( $122 \mathrm{meV}) .{ }^{29}$ The progression in $v_{2}$, however, falls off in intensity much more rapidly in the cation than in the anion. As noted by others previously ${ }^{3(\mathrm{c}), 3(\mathrm{~d})}$ the anion progression is rather anharmonic.

The midpoint of the first vibrational structure occurs at $1.12 \pm 0.03 \mathrm{eV}$. Since this feature has the largest excursion from the maximum to the minimum in the derivative, we assign this value to the vertical attachment energy, superceding our measurement of $1.15 \pm 0.03 \mathrm{eV}$ reported earlier. ${ }^{6(\mathrm{a})}$ A table of previous experimental results and theoretical values is given by Nenner and Schulz. ${ }^{3(d)}$ We expect that the adiabatic attachment energy corresponds closely to the vertical value since the distortion of the benzene molecule upon attachment of the electron should be relatively small. Minimal basis set calculations of Hinde $e t$ al.$^{30}$ indicate that both the ${ }^{2} A_{u}$ and ${ }^{2} B_{1 u}$ states resulting from $D_{2 h}$ distortion lie about $0.2 \mathrm{eV}$ below the energy of the anion with the geometry of the neutral molecule. Larger basis sets containing more diffuse functions might give smaller geometry distortions.

In view of the relatively sharp structure in the ET spectrum, and hence moderately long lifetime of the anion, one might expect that structure due to the $v_{16}\left(e_{2 g}\right) \mathrm{Jahn}$-Teller mode, reflecting the motion toward the relaxed configuration, would be visible. No evidence for this is observed in the ET spectrum. In contrast, the first photoelectron band of benzene shows stronger excitation of the $v_{16} \mathrm{Jahn}-\mathrm{Teller}$ mode than $v_{2}{ }^{29}$ Structure due to the $v_{17}$ and $v_{18} \mathrm{Jahn}$-Teller modes is also evident in the photoelectron spectrum. The work of Wong and Schulz, ${ }^{24}$ however, shows that vibrational excitation of neutral benzene through the intermediate formation of $\mathrm{C}_{6} \mathrm{H}_{6}-\left({ }^{2} E_{2 u}\right)$ excites one quantum of $v_{16}\left(e_{2 g}\right)$ with excitation of multiple levels of $v_{2}\left(a_{1 g}\right)$, the mode dominating the ET spectrum. Excitation of a single quantum of $v_{20}\left(e_{2 u}\right)$, a ring puckering mode, also occurs together with $n v_{2}$. Calculations of Lipari et al. ${ }^{31}$ suggest that the $v_{17}$ and $v_{18}$ Jahn-Teller modes should also be excited in the anion. That such excitations do not appear in the energy loss measurement may be due to the smaller coupling constant ${ }^{31}$ of the former and the lower frequency of vibration of the latter relative to that of $v_{16}$.

Weak excitation of $v_{20}$ through the resonance, as observed in energy loss, implies that the anion can lower its energy by distorting out-of-plane. It is important to note that this does not necessarily imply that the anion is nonplanar in its equilibrium structure. In fact the calculations of Hinde 
TABLE I. Vertical electron affinities $(\mathrm{eV}) \mathrm{e}^{\mathrm{a}, \mathrm{b}}$

\begin{tabular}{|c|c|c|c|c|}
\hline Compound & Anion state & ETS & PPP & $\mathrm{HAM} / 3$ \\
\hline \multirow[t]{4}{*}{ Benzene } & ${ }^{2} E_{2 u}\left(\pi_{1}^{*}, \pi_{2}^{*}\right)$ & -1.12 & -1.07 & -1.11 \\
\hline & ${ }^{2} B_{2 g}\left(\pi_{3}^{*}\right)$ & -4.82 & -3.97 & -4.86 \\
\hline & $2 p-1 h$ & -5.7 & & \\
\hline & $2 p-1 h$ & -7.5 & & \\
\hline \multirow[t]{11}{*}{ Naphthalene } & ${ }^{2} B_{2 g}\left(\pi_{1}^{*}\right)$ & -0.19 & -0.06 & +0.36 \\
\hline & ${ }^{2} B_{1 g}\left(\pi_{2}^{*}\right)$ & -0.90 & -0.78 & -0.49 \\
\hline & ${ }^{2} B_{3 u}\left(\pi_{3}^{*}\right)$ & -1.67 & -1.69 & -1.68 \\
\hline & ${ }^{2} A_{u}\left(\pi_{4}^{*}+2 \mathrm{p}-1 \mathrm{~h}\right)$ & $\sim-2.5$ & & \\
\hline & $\left.{ }^{2} A_{u}\left(\pi_{4}^{*}+2 \mathrm{p}-1 \mathrm{~h}\right)\right)$ & & -2.66 & -2.94 \\
\hline & & -3.38 & & \\
\hline & ${ }^{2} B_{1 \mathrm{~g}}\left(\pi_{5}^{*}+2 \mathrm{p}-1 \mathrm{~h}\right)$ & & -4.29 & -5.60 \\
\hline & ${ }^{2} B_{\mathrm{lg}}\left(\pi_{\xi}^{*}+2 \mathrm{p}-1 \mathrm{~h}\right)$ & -4.72 & & \\
\hline & $2 p-1 h$ & -5.67 & & \\
\hline & $2 p-1 h$ & -7.10 & & \\
\hline & $2 \mathrm{p}-1 \mathrm{~h}$ & -8.72 & & \\
\hline \multirow[t]{12}{*}{ Anthracene } & ${ }^{2} B_{3 u}\left(\pi_{1}^{*}\right)$ & $>0$ & +0.49 & +1.31 \\
\hline & ${ }^{2} A_{4}(\pi)$ & -0.60 & -0.65 & -0.16 \\
\hline & ${ }^{2} B_{2 g}\left(\pi_{3}^{*}\right)$ & -1.13 & -0.92 & -0.33 \\
\hline & ${ }^{2} B_{34}\left(\pi_{4}^{*}\right)$ & -2.15 & -1.92 & -1.69 \\
\hline & ${ }^{2} B_{1 g}\left(\pi \pi_{5}^{*}+2 \mathrm{p}-1 \mathrm{~h}\right)$ & -1.62 & & \\
\hline & $\left.{ }^{2} B_{1 g}\left(\pi_{5}^{*}+2 \mathrm{p}-1 \mathrm{~h}\right)\right)$ & & -1.98 & -1.84 \\
\hline & & -2.63 & & \\
\hline & ${ }^{2} A_{u}\left(\pi_{6}^{*}+2 \mathrm{p}-1 \mathrm{~h}\right)$ & & -3.39 & -3.90 \\
\hline & & -3.57 & & \\
\hline & $\left.{ }^{2} B_{1 g}\left(\pi_{7}^{*}+2 \mathrm{p}-1 \mathrm{~h}\right)\right\}$ & & -4.37 & -5.58 \\
\hline & ${ }^{2} B_{1 g}\left(\pi_{7}^{*}+2 \mathrm{p}-1 \mathrm{~h}\right)$ & -4.50 & & \\
\hline & $2 \mathrm{p}-1 \mathrm{~h}$ & -6.05 & . & \\
\hline \multirow[t]{7}{*}{ Tetracene } & ${ }^{2} B_{2 g}\left(\pi_{i}^{*}\right)$ & $>0$ & +0.84 & +1.80 \\
\hline & ${ }^{2} B_{3 u}\left(\pi_{2}^{*}\right),{ }^{2} B_{1 g}\left(\pi_{3}^{*}\right)$ & -0.40 & $-0.36,-0.58$ & $+0.46,+0.17$ \\
\hline & ${ }^{2} B_{2 g}\left(\pi_{4}^{*}\right),{ }^{2} A_{u}\left(\pi_{5}^{*}\right)$ & -1.25 & & $-0.78,-1.00$ \\
\hline & ${ }^{2} B_{3 \mu}\left(\pi_{6}^{*}\right)$ & -1.93 & & -1.70 \\
\hline & ${ }^{2} B_{1 g}\left(\pi \pi_{7}^{*}\right)$ & $\sim-2.95$ & & -2.76 \\
\hline & ${ }^{2} A_{u}\left(\pi_{8}^{*}\right)$ & -3.45 & & -4.51 \\
\hline & ${ }^{2} B_{1 g}\left(\pi_{9}^{*}\right)$ & -4.85 & & -5.60 \\
\hline \multirow[t]{7}{*}{ Styrene $\left(C_{2 v}\right)$} & ${ }^{2} B_{1}\left(\pi_{1}^{*}\right)$ & -0.24 & -0.39 & +0.13 \\
\hline & ${ }^{2} A_{2}\left(\pi_{2}^{*}\right)$ & -1.04 & -1.07 & -0.81 \\
\hline & ${ }^{2} B_{1}\left(\pi_{3}^{*}\right)$ & -2.49 & -2.27 & -2.19 \\
\hline & ${ }^{2} B_{1}\left(\pi_{4}^{*}+2 \mathrm{p}-1 \mathrm{~h}\right)$ & $\sim-3.6$ & & \\
\hline & & & -4.21 & -4.87 \\
\hline & ${ }^{2} B_{1}\left(\pi_{4}^{*}+2 \mathrm{p}-1 \mathrm{~h}\right)$ & -4.67 & & \\
\hline & $2 p-1 h$ & -5.7 & & \\
\hline Trans-stilbene & ${ }^{2} B_{g}\left(\pi_{1}^{*}\right)$ & $>0$ & +0.15 & +1.15 \\
\hline \multirow{5}{*}{$\left(C_{2 h}\right)$} & ${ }^{2} A_{u},{ }^{2} B_{g}\left(\pi_{2,3}^{*}\right)$ & -0.86 & $-0.96,-0.96$ & $-0.15,-0.15$ \\
\hline & ${ }^{2} A_{u}\left(\pi_{4}^{*}\right)$ & -1.43 & -1.18 & -0.47 \\
\hline & ${ }^{2} B_{g}\left(\pi_{5}^{*}\right)$ & -2.56 & -2.29 & -1.97 \\
\hline & ${ }^{2} A_{u}\left(\pi_{6}^{*}\right)$ & -4.37 & -3.77 & -3.96 \\
\hline & ${ }^{2} B_{g}\left(\pi_{7}^{*}\right)$ & $\sim-5.03$ & -4.08 & -4.56 \\
\hline \multirow{6}{*}{ Cis-stilbene $\left(C_{2 v}\right)$} & ${ }^{2} A_{2}\left(\pi_{1}^{*}\right)$ & $>0$ & & \\
\hline & ${ }^{2} B_{1},{ }^{2} A_{2}\left(\pi_{2,3}^{*}\right)$ & -0.79 & & \\
\hline & ${ }^{2} B_{1}\left(\pi_{4}^{*}\right)$ & -1.25 & & \\
\hline & ${ }^{2} A_{2}(\pi)$ & -2.50 & & \\
\hline & ${ }^{2} B_{1}\left(\pi_{6}^{*}\right)$ & -4.52 & & \\
\hline & ${ }^{2} A_{2}\left(\pi_{7}^{*}\right)$ & $?$ & & \\
\hline
\end{tabular}

Error limits for the ET structures are $\pm 0.03 \mathrm{eV}$ for the narrow features and refer to measurements under high rejection conditions. Broad features are located to $\pm 0.05 \mathrm{eV}$ or in a few cases $\pm 0.1 \mathrm{eV}$. Note that for a single structure attributed to two unresolved resonances, the resonance energies may be more uncertain.

${ }^{b}$ For naphthalene and the larger acenes, the symmetry labels are derived assuming that the molecules lie in the $y z$ plane with $z$ on the long molecular axis. 
et $a .^{30}$ and ESR studies ${ }^{32}$ indicate that the anion in its most stable configuration is planar. Thus, it appears that upon vertical attachment of an electron, the resulting $\mathrm{C}_{6} \mathrm{H}_{6}$ anion can lower its energy by any of three distortions; (i) enlargement of the ring $\left(v_{2}\right)$, (ii) out-of-plane distortion $\left(v_{20}\right)$, or (iii) Jahn-Teller distortion $\left(v_{16}\right)$, all of which decrease the antibonding nature in the $\pi^{*}$ orbital. Even though the anion as initially formed can lower its energy by distorting out-of-plane, the overall minimum corresponds to a $D_{2 h}$ Jahn-Teller distorted species. In this configuration the antibonding nature of the $\pi^{*}$ orbitals is apparently diminished sufficiently that further energy lowering by nonplanar distortion is not possible.

Turning now to the higher lying resonances, curve (b) in Fig. 2 shows the transmission spectrum of benzene from just below the $4.82 \mathrm{eV}$ resonance upward to $14 \mathrm{eV}$. This spectrum was taken at higher gas density and greater beam current than usual and reveals features not previously reported using the transmission method.

Assignment of the $4.82 \mathrm{eV}$ resonance to $\mathrm{a}^{2} B_{2 g}$ tempo-

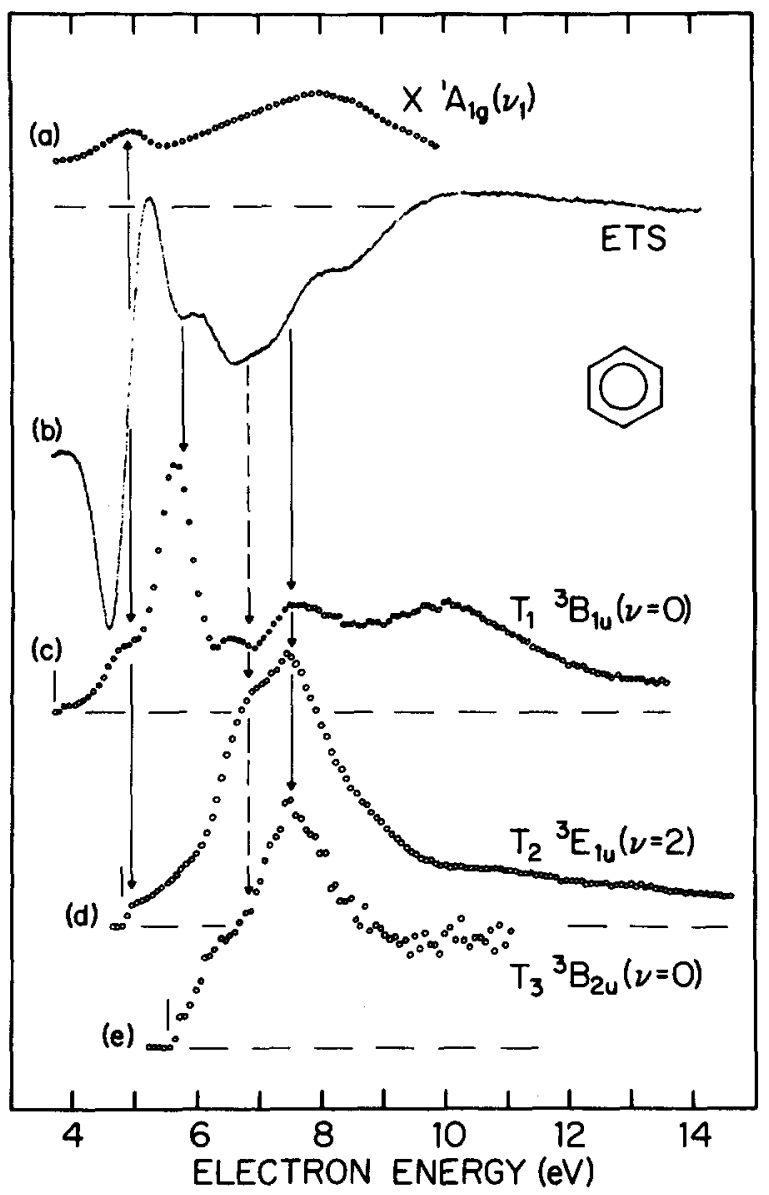

FIG. 2. Decay channels of resonances in benzene. (a) The excitation function for the $v_{1}$ mode of the ground electronic state of benzene (Ref. 33). (b) The electron transmission spectrum over the range 4-14 eV. (c), (d), (e) The excitation functions of the three lowest triplet states of benzene (Ref. 23). The vertical lines are a guide associating the structure in the ET spectrum with that in the excitation functions. rary anion state has been confirmed by the angular distribution analysis in the electron scattering experiments of Wong and Schulz. ${ }^{24}$ This resonance, of nominally "shape" or single particle character, is expected to decay primarily into the vibrational levels of the "parent" state, namely, the ground electronic state of benzene. This was demonstrated by Azria and Schulz ${ }^{33}$ whose data for the excitation of the $v_{1}$ mode of the benzene ground state is shown in curve (a). Note that the midpoint energies in the resonance profiles of curve (b) should correspond closely to the energies of maxima in the excitation functions. The vertical arrows originating on the ETS curve serve to guide the eye in Fig. 2 for this correspondence. There is good agreement between the $4.9 \mathrm{eV}$ maximum in curve (a) and the position of the ${ }^{2} B_{2 g}$ resonance. The width of the peak in the excitation function, furthermore, agrees well with the spacing between the extrema in the derivative spectrum.

Nenner and Schulz ${ }^{3(d)}$ have pointed out that the 4.82 $\mathrm{eV}^{2} B_{28}$ resonance may also contain an admixture of twoparticle, one-core hole $(2 \mathrm{p}-1 \mathrm{~h})$ configurations along with the dominant $\left(a_{2 u}\right)^{2}\left(e_{1 g}\right)^{4} b_{2 g}$ one-particle (1p) configuration. Their assertion was based on the close proximity of neutral excited electronic states of configuration $\left(a_{2 u}\right)^{2}$ $\left(e_{1 g}\right)^{3} e_{2 u}$, and on the observation that an additional electron in the lowest $e_{2 u}$ unfilled orbital yields anion states of ${ }^{2} B_{2 g}$ symmetry, among others. More directly, Azria and Schulz ${ }^{33}$ observed that the resonance, upon ejection of an electron, also decays into the ${ }^{3} B_{1 u}(3.66 \mathrm{eV}),{ }^{3} E_{1 u}(4.51 \mathrm{eV})$, and ${ }^{1} B_{2 u}$ $(4.72 \mathrm{eV})$ states, all of which arise from the configuration above. To illustrate this more clearly we show in curves (c), (d), and (e) of Fig. 2, the excitation functions of the three lowest triplet states of benzene measured very recently by Allan. ${ }^{23}$ Excitation of $T_{1}\left({ }^{3} B_{1 u}\right)$ in (c) exhibits a clear-cut shoulder just above its threshold. Excitation to the $v_{2}=2$ level of $T_{2}\left({ }^{3} E_{1 u}\right)$ in (d) starts abruptly at its threshold, which is also suggestive of production through the ${ }^{2} B_{2 g}$ resonance. These data demonstrate that a single-particle description of this resonance, as an electron bound to the molecule in its ground electronic state, is incomplete, and that the effects of configuration mixing must be considered.

The transmission spectrum, curve (b) in Fig. 2, shows evidence for a number of other resonances above $5 \mathrm{eV}$ which must be made up primarily of $2 \mathrm{p}-1 \mathrm{~h}$ configurations. These resonances are overlapping and the midpoints cannot be determined accurately; nevertheless, there is good correspondence between the energies and widths of these structures and the peaks in the triplet excitation functions measured by Allan. ${ }^{23}$ The most prominent resonances lie near 5.7 and 7.5 $\mathrm{eV}$. It is likely that these resonances are due to the five doublet states $\left(1^{2} E_{1 g}, 2^{2} E_{1 g}, 3^{2} E_{1 g},{ }^{2} B_{1 g}\right.$, and $\left.{ }^{2} B_{1 g}\right)$ derived from the $e_{1 g}^{3} e_{2 u}^{2}$ configuration.

Finally, returning to excitation of the vibrational levels of the ground electronic state, curve (a) displays a broad enhancement centered at $8.0 \mathrm{eV}$. The half-width of this peak is too great to correspond to any of the resonances discussed earlier, although there is a broad variation in the transmitted signal between 6.6 and $10 \mathrm{eV}$ which may be related. A possible assignment would be to one or more shape resonances associated with unfilled $\sigma^{*}$ orbitals. 


\section{Naphthalene}

An overall view of the transmission spectrum of naphthalene between 0 and $10 \mathrm{eV}$ is given in curves (a) and (b) of Fig. 3. Allowing for the widths of the resonances, this figure indicates that there is virtually no impact energy below $10 \mathrm{eV}$ at which electrons are not temporarily trapped. An expanded view showing the vibrational structure in the two lowest anion states is presented in Fig. 4.

Low energy electron scattering in naphthalene has been carried out using a number of techniques. The second resonance was first observed using the $\mathrm{SF}_{6}$ scavenger technique by Compton et al..$^{34}$ who reported structure at $0.8 \mathrm{eV}$. Somewhat later Pisanias et al., ${ }^{35}$ using the trapped electron method, observed two resonances at 0.75 and $1.3 \mathrm{eV}$. The lowest resonance was first reported at $0.20 \pm 0.50 \mathrm{eV}$, using ETS, by Nenner and Schulz ${ }^{3(d)}$ but no discussion or spectrum was given. Mathur and Hasted ${ }^{36}$ presented a more comprehensive list of resonances measured in transmission using an electrostatically collimated electron beam without the derivative scheme. Our energies for the lowest five resonances were originally listed in a short review ${ }^{2}$ in 1978 without the spectrum. Finally, Allan ${ }^{37}$ has recently carried out a transmission measurement in a magnetically collimated system but without use of the derivative method. The results from all the experimental studies are summarized in Table II.

The data of Mathur and Hasted ${ }^{36}$ are only in fair agreement with the present results for the lowest three resonances, with two of these falling outside the range of quoted errors.

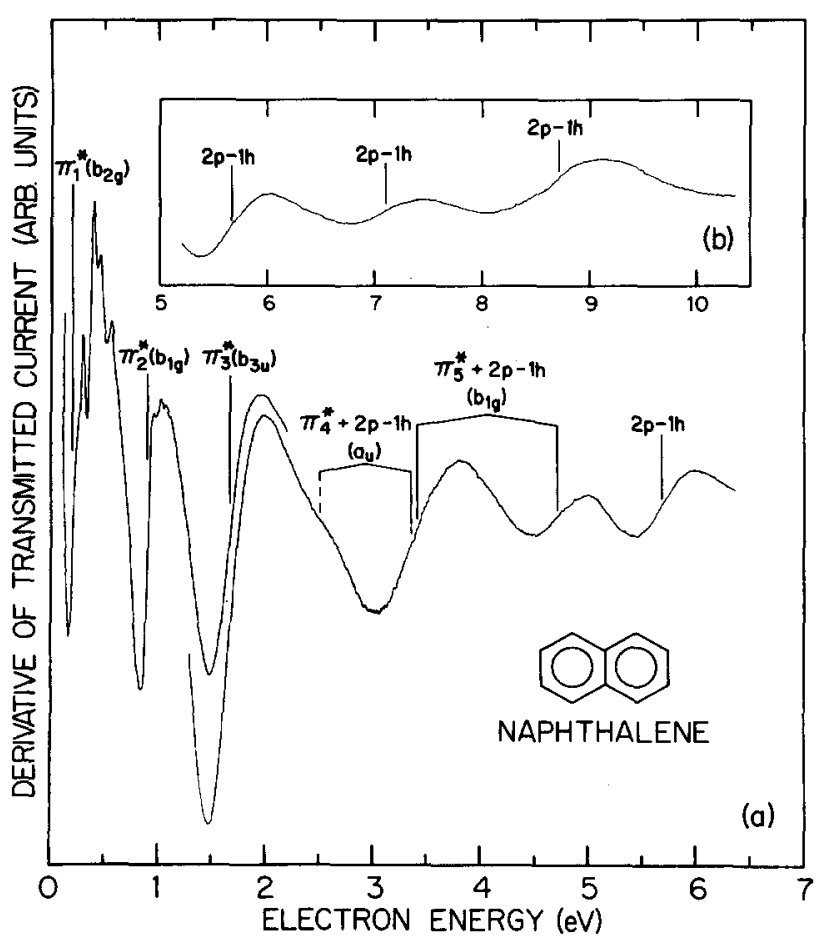

FIG. 3. The electron transmission spectrum of naphthalene. The anion states which are primarily single particle in nature are labeled with the appropriate $\pi^{*}$ orbital and symmetry. The broad feature centered at $3.38 \mathrm{eV}$ is attributed to two overlapping unresolved contributions from $\pi_{4}^{*}$ and $\pi_{5}^{*}$ each mixed with the two-particle, one-hole configuration of proper symmetry.

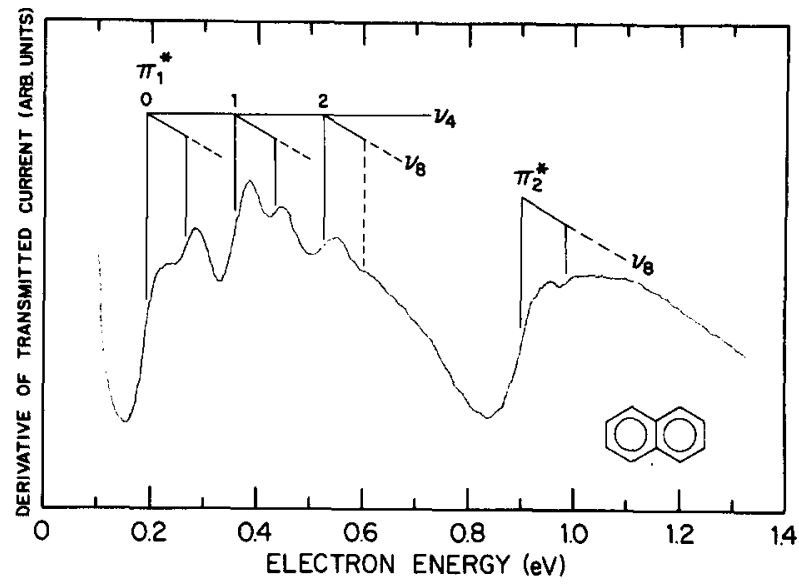

FIG. 4. Expanded view of the transmission spectrum of naphthalene showing the first two anion states.

The upper two resonances in their paper are in more substantial disagreement, and the two intervening features at 3.38 and $4.72 \mathrm{eV}$ were not reported. Allan's measurements below $6 \mathrm{eV}$, however, are in excellent agreement with our data. The resonance at $8.72 \mathrm{eV}$ is not readily discernible in his spectrum.

As observed in Fig. 4, the lowest resonance displays pronounced vibrational structure, contrary to the results of Mathur and Hasted who concluded that either the anion lifetimes were too short to permit appreciable nuclear motion or that the anion curves are repulsive in the FranckCondon region. Repeated measurements in several ET instruments under different operating conditions have consistently shown these features. The second resonance shows only faint evidence of nuclear motion.

The structure in the lowest anion state arises from both high and low frequency modes. The spacing between successive members of the high frequency progression is 170 and $165 \mathrm{meV}$. The most likely candidate for this mode is $v_{4}\left(a_{8}\right)$ which is also strongly excited in the photoelectron spectrum and has a spacing of $170 \mathrm{meV}$ in the ground state cation ${ }^{38}$ and $181 \mathrm{meV}$ in the ground state of the neutral molecule. ${ }^{39}$ The low frequency mode, which has a spacing of approximately $78 \mathrm{meV}$ in the ET spectrum, is most reasonably assigned to $v_{8}, 94 \mathrm{meV}$ in the neutral. ${ }^{39}$ The only remaining totally symmetric vibration in the neutral molecule is $v_{9}$ at

TABLE II. Attachment energies in naphthalene (eV).

\begin{tabular}{cccccc}
\hline \hline $\begin{array}{c}\text { Present } \\
\text { data }\end{array}$ & $\begin{array}{c}\text { Mathur and } \\
\text { Hasted } \\
\text { (Ref. 21) }\end{array}$ & $\begin{array}{c}\text { Allan } \\
\text { (Ref. 23) }\end{array}$ & $\begin{array}{c}\text { Nenner } \\
\text { and Schulz } \\
\text { [Ref. (3d)] }\end{array}$ & $\begin{array}{c}\text { Compton } \\
\text { et al. } \\
\text { (Ref. 34) }\end{array}$ & $\begin{array}{c}\text { Pisanias } \\
\text { et al. } \\
\text { (Ref. 35) }\end{array}$ \\
\hline 0.19 & $0.20 \pm 0.05$ & & $0.20 \pm 0.05$ & & \\
0.90 & $0.76 \pm 0.05$ & $\sim 0.8$ & & 0.8 & 0.75 \\
1.67 & $0.35 \pm 0.08$ & $\sim 1.7$ & & & 1.3 \\
$\sim 2.5$ & & & & & \\
3.38 & & $\sim 3.3$ & & \\
4.72 & & $\sim 4.7$ & & \\
5.67 & $5.29 \pm 0.1$ & $\sim 5.7$ & & \\
7.10 & $7.77 \pm 0.05$ & $\sim 7.3$ & & \\
8.72 & & & & \\
\hline \hline
\end{tabular}


$64 \mathrm{meV}$ which seems rather low. The vibrational structure in the second resonance is very weak and is characterized by a spacing of about $85 \mathrm{meV}$, approximately that of the $v_{8}$ mode.

As discussed in more detail below, the resonance observed in the energy range below $0.7 \mathrm{eV}$ is undoubtedly the ground electronic state of the anion. A determination of the vertical attachment energy to this state is therefore of considerable interest. The essential question is thus whether the feature at $0.19 \mathrm{eV}$ is the lowest vibrational level of the observed progression. There is the possibility that lower vibrational levels are not seen either because they overlap the main unscattered electron beam or because the anion lifetime is too long to allow detection of the anion in a scattering experiment.

Although this question cannot be resolved with complete certainty, the evidence suggests that the vertical EA is indeed $-0.19 \mathrm{eV}$. If there were a lower level of the $170 \mathrm{meV}$ progression, it would fall at an impact energy between 15 and $20 \mathrm{meV}$ and would be completely obscured by the main electron beam. However, since each member of the $v_{4}$ progression seen in the ET spectrum is accompanied by a low frequency excitation lying $78 \mathrm{meV}$ higher, it is not unreasonable to expect a feature near $90-95 \mathrm{meV}$. At this energy there is still a substantial wing to the electron energy distribution, however, a diligent search under a range of experimental conditions did not reveal evidence for an additional feature. A low lying resonance could in principle have such a narrow width that detection would be difficult. The leading term in a Legendre polynomial expansion ${ }^{40}$ of the lowest unoccupied orbital of naphthalene corresponds to an angular momentum of $l=2$ so a substantial barrier should exist. Nevertheless, given the sizes and widths of the observed features, it is unlikely that a somewhat enhanced lifetime would result in complete suppression of a feature near $90-95 \mathrm{meV}$.

From simple molecular orbital considerations one expects similar distortions of the molecule upon electron attachment into the lowest unoccupied molecular orbital and upon ionization from the highest occupied molecular orbital and hence the same length vibrational progression in each of the respective spectra. The transmission spectrum of naphthalene reveals three members in the ring breathing mode, consistent with the appearance of three pronounced members in the first photoelectron band, and again this supports our assignment of the EA.

Assuming the validity of the pairing theorem, one can use the first two IP's and the second EA to predict the first EA. This procedure gives rise to a value of $-0.19 \mathrm{eV}$ in naphthalene, in excellent agreement with the first peak seen in the ET spectrum. In other alternant hydrocarbons with two low lying anion states (e.g., styrene and stilbene) and for which appreciable configuration mixing should not occur in either the anion or cation states, the errors in the EA's predicted in such a manner are quite small.

The semiempirical PPP calculations of Younkin et al. ${ }^{41}$ yield a vertical EA of $-0.06 \mathrm{eV}$. However, the second anion state is predicted at $-0.78 \mathrm{eV}$ which is too positive by 0.12 $\mathrm{eV}$. The separation between the first two anion states is in excellent agreement with our data. The HAM/3 method of Åsbrink, Lindholm, and co-workers ${ }^{42}$ yields a first EA for naphthalene of $+0.36 \mathrm{eV}$. However, Åsbrink et al. ${ }^{43}$ arrive at a preferred EA of $\approx 0.0 \mathrm{eV}$ after applying corrections of $0.4 \mathrm{eV}$ to some, but not all, orbitals to force better agreement with the PES, ETS, and anion optical absorption spectra. It is not clear that this correction can be done with sufficient accuracy to distinguish the result from our suggested value.

Finally, Wentworth et al. ${ }^{44}$ who studied the attachment of thermalized electrons to naphthalene in the high pressure environment used in gas chromatographic detectors, have inferred an electron affinity of $+0.15 \mathrm{eV}$. However, we are not aware of any beam studies which support the existence of a stable anion of naphthalene in the gas phase. Christophorou and Chaney ${ }^{43}$ indicate a very low electron attachment rate in swarm experiments and further point out that the rate should be treated as an upper bound because of the possibility that it may be influenced by impurities.

We now address the state assignments for the resonances observed in Fig. 3. In the absence of configurational mixing, five low lying negative ion states, one associated with each of the normally unoccupied $\pi^{*}$ orbitals, would be expected in naphthalene. In marked contrast to the ET results in benzene where the predominately $1 p$ shape resonances are substantially larger than those features we attribute to $2 p-1 h$ anion states, the naphthalene spectrum offers no clear guide permitting us to separate $1 \mathrm{p}$ and $2 \mathrm{p}-1 \mathrm{~h}$ resonances.

In an earlier look at this problem, ${ }^{11}$ it was our contention that the features in the ET spectrum, particularly at higher energies, did not reveal a simple correspondence to the energies calculated using "standard" CI methods or to the absorption spectra of the ground state anions in condensed glasses. Many of the higher lying anion states will have considerable $2 \mathrm{p}-1 \mathrm{~h}$ character and are expected to be weak in ETS. Those $2 \mathrm{p}-1 \mathrm{~h}$ states which can be formed by a $\pi \rightarrow \pi^{*}$ excitation from the $1 \mathrm{p}$ ground state can appear strongly in the optical absorption spectra if they are dipole allowed. On the other hand, the $2 \mathrm{p}-1 \mathrm{~h}$ and $1 \mathrm{p}$ states which are dipole forbidden will not appear or will appear weakly due to vibronic coupling. There is ample room therefore for significant differences in the respective spectroscopies.

In interpreting the ET spectra of the acenes it is useful to make a more direct comparison of the gas phase data with that from solution studies as well as with the predictions from CI calculations and the single particle methods. In Fig. 5 we display this information, plotting the optical absorption spectrum of the naphthalene anion as measured by Shida and Iwata. ${ }^{46}$ The zero in the optical transition energy is shifted to lie at the ground state of the anion so that the alignment of the absorption maxima with the temporary anion energies is more evident. We have taken an EA of $-0.19 \mathrm{eV}$ for this purpose. Along with the ETS energies, we also give the theoretical predictions of $\mathrm{PPP}^{41}, \mathrm{HAM} / 3^{42}$ and $\mathrm{CI}^{47}$ calculations. The CI results of Zahradnik and Carsky ${ }^{47}$ were chosen in preference to those computed by Shida and Iwata ${ }^{46}$ because the former authors included several optically forbidden transitions.

Before proceeding with a detailed discussion of naphthalene it is useful to note a few generalizations which can be made from this comparison and those for anthracene and 


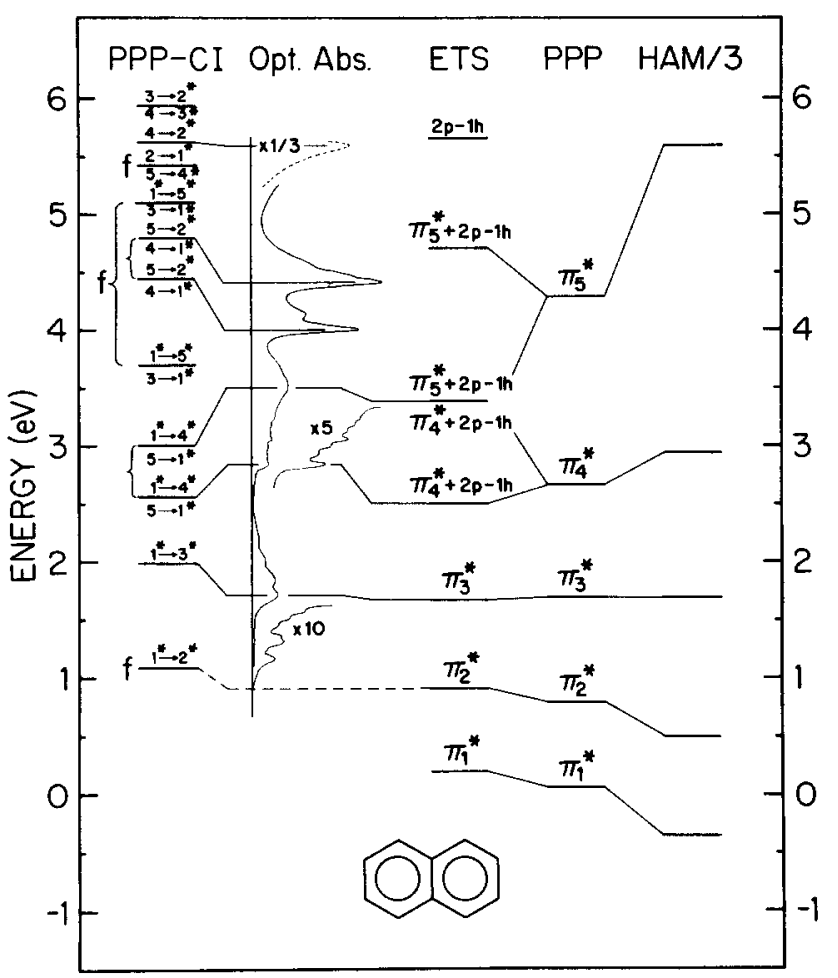

FIG. 5. A comparison of naphthalene anion energies derived from ETS, optical absorption measurements on the anion in solution (Ref. 46), and PPP (Ref. 41) and HAM/3 (Ref. 42) calculations. The energy scale for the optical absorption data has been shifted so that its zero lies at $+0.19 \mathrm{eV}$ in agreement with ETS. On the left, energies from PPP-CI calculations (Ref. 47) are shown along with the major contributing configurations. The $\pi$ orbitals are abbreviated by $1-5$ (bonding) and $1^{*}-5^{*}$ (antibonding). Optically forbidden transitions from the anion ground state are labeled by " $f$." States of the same configuration are connected with brackets.

tetracene shown in Figs. 7 and 9.

The optical absorption spectra are dominated by three intense, narrow peaks, at 5.5, 4.4, and $4.0 \mathrm{eV}$ in naphthalene using the energy scale in Fig. 5 . These features maintain their shapes and relative spacings as they shift to lower energies in anthracene and tetracene. These optical transitions, which are polarized along the long axis of the molecules, appear to have no counterpart in the ET spectra. The CI calculations ${ }^{46,47}$ show that they are almost entirely $2 \mathrm{p}-1 \mathrm{~h}$ in nature and dominated in the naphthalene anion by configurations which involve $\pi_{4} \rightarrow \pi_{1}^{*}$ and $\pi_{5} \rightarrow \pi_{2}^{*}$ excitations from the $\cdots \pi_{4}^{2} \pi_{5}^{2} \pi_{1}^{*}$ ground state. In anthracene and tetracene, the analogous contributions are $\pi_{6} \rightarrow \pi_{1}^{*}, \pi_{7} \rightarrow \pi_{2}^{*}$ and $\pi_{8} \rightarrow \pi_{1}^{*}, \pi_{9} \rightarrow \pi_{2}^{*}$, respectively. The optical transitions are thus ${ }^{2} B_{2 g} \rightarrow{ }^{2} B_{3 u}$ in the naphthalene and tetracene anions and ${ }^{2} B_{3 u} \rightarrow{ }^{2} B_{2 g}$ in the anthracene anion.

The lowest-lying $2 \mathrm{p}-1 \mathrm{~h}$ configuration is derived from the ground state anion by exciting an electron from the highest occupied $\pi$ to the partially filled lowest $\pi^{*}$ orbital. This is a dipole allowed transition giving an excited state of ${ }^{2} A_{u}$ symmetry in the naphthalene and tetracene anions and ${ }^{2} B_{1 g}$ in that of anthracene. On our energy scale the unmixed $2 \mathrm{p}-$ 1h configurations fall roughly at $3,2.3$, and $1.4 \mathrm{eV}$ in naphthalene, anthracene, and tetracene, respectively. These values are estimated from comparison of the theoretical and condensed phase transition energies. This places these $2 \mathrm{p}-1 \mathrm{~h}$ configurations energetically close to the $1 \mathrm{p}$ configurations of the same symmetry, giving rise to appreciable mixing. The lowest lying $2 \mathrm{p}-1 \mathrm{~h}$ configuration which is dipole forbidden with respect to the ground state anion is expected to lie considerably $(\approx 1 \mathrm{eV}$ ) above the above-mentioned dipole allowed $2 p-1$ configurations. These considerations lead us to conclude that the first three $1 p$ anion levels will undergo relatively little mixing with $2 \mathrm{p}-1 \mathrm{~h}$ configurations in these compounds. This is in contrast to the linear polyenes for which appreciable configuration mixing is expected in the first excited anion state. ${ }^{\mathbf{4 8 , 4 9}}$

Finally, the lowest transitions derived from promoting an electron from $\pi_{1}^{*}$ to $\pi_{2}^{*}$ in naphthalene and anthracene and from $\pi_{1}^{*}$ to $\pi_{3}^{*}$ in tetracene, $\pi_{2}^{*}$ and $\pi_{3}^{*}$ lying close together in this latter compound, are optically forbidden. The optical spectra however show weak structure due to vibronic activity $^{46}$ above the threshold excitation in all three compounds.

With this background, we turn now to complete our discussion of the ET spectrum of naphthalene. In Fig. 3 we have indicated above each resonance our preferred assignment. Those states which are expected to have considerable $2 \mathrm{p}-1 \mathrm{~h}$ character, based on the CI calculations, are so indicated.

(a) The energies of the first three resonances agree closely with the PPP predictions. As argued above, $\mathrm{CI}$ is not expected to be a consideration in these states, and the assignments follow in a straightforward manner.

(b) The resonances at 3.38 and $4.72 \mathrm{eV}$ lie $0.4-0.7 \mathrm{eV}$ above the PPP predictions. Furthermore the $3.38 \mathrm{eV}$ feature is broader than the neighboring resonances, and there is a weak "shoulder" lying near $2.5 \mathrm{eV}$. As noted earlier, the CI calculations ${ }^{46,47}$ indicate that the lowest ${ }^{2} A_{u}$ and ${ }^{2} B_{1 \mathrm{~g}} 2 \mathrm{p}-1 \mathrm{~h}$ configurations lie close to those of the $a_{u}$ and $b_{1 g} \pi^{*}$ orbitals. Based on the calculated splitting, we suggest that the upper member of the ${ }^{2} A_{u}$ resonance and the lower of the ${ }^{2} B_{1 g}$ fall under the broad $3.38 \mathrm{eV}$ resonance. The lower ${ }^{2} A_{u}$ is thus attributed to the weak shoulder at $2.5 \mathrm{eV}$ and the upper ${ }^{2} B_{18}$ to the feature at $4.72 \mathrm{eV}$. Although the various CI calculations agree in demonstrating appreciable mixing in this energy range, we note that they show sizable differences in the degree of mixing.

(c) The features above $5 \mathrm{eV}$ are undoubtedly mostly $2 \mathrm{p}-$ $1 \mathrm{~h}$ in character. The resonance at $8.72 \mathrm{eV}$ warrants further study as it lies about $0.6 \mathrm{eV}$ above the first ionization potential and thus is unstable with respect to detachment of two electrons.

\section{Anthracene}

Figure 6 shows the ET spectrum below $7 \mathrm{eV}$, and Fig. 7 summarizes the ETS, HAM, PPP, and optical absorption energies. The energy scale of the optical absorption spectrum is shifted to correspond to a ground state anion at $-0.5 \mathrm{eV}$, the anthracene first EA of $+0.5 \mathrm{eV}$ being derived from the pairing theorem. This value is also in excellent agreement with that predicted by the PPP calculations. The energies of the first two features in the ET spectrum are in good agreement with the PPP energies for the second $\left({ }^{2} A_{u}\right)$ and third $\left({ }^{2} B_{2 g}\right)$ anion states. These assignments are also 


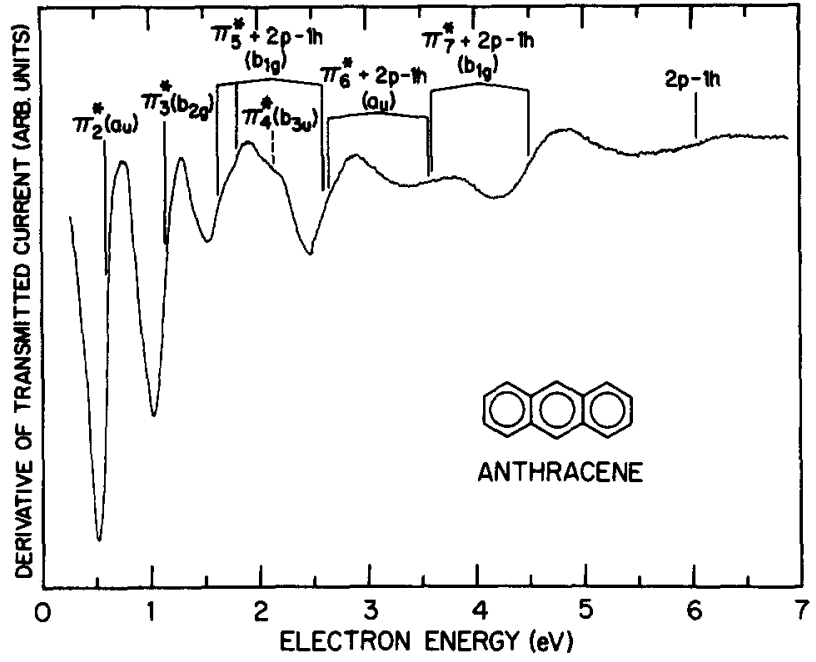

FIG. 6. Electron transmission spectrum of anthracene. The features centered at 2.63 and $3.57 \mathrm{eV}$ are each attributed to two overlapping unresolved resonances.

consistent with the energies and intensities of the first two features seen in the absorption spectra of the ground state anion.

Close examination of the third major feature in the ET spectrum, centered at $1.67 \mathrm{eV}$, reveals that it consists of two closely spaced structures at 1.62 and $1.80 \mathrm{eV}$ and a third shoulder at $2.15 \mathrm{eV}$. Each of the features falls within $50 \mathrm{meV}$

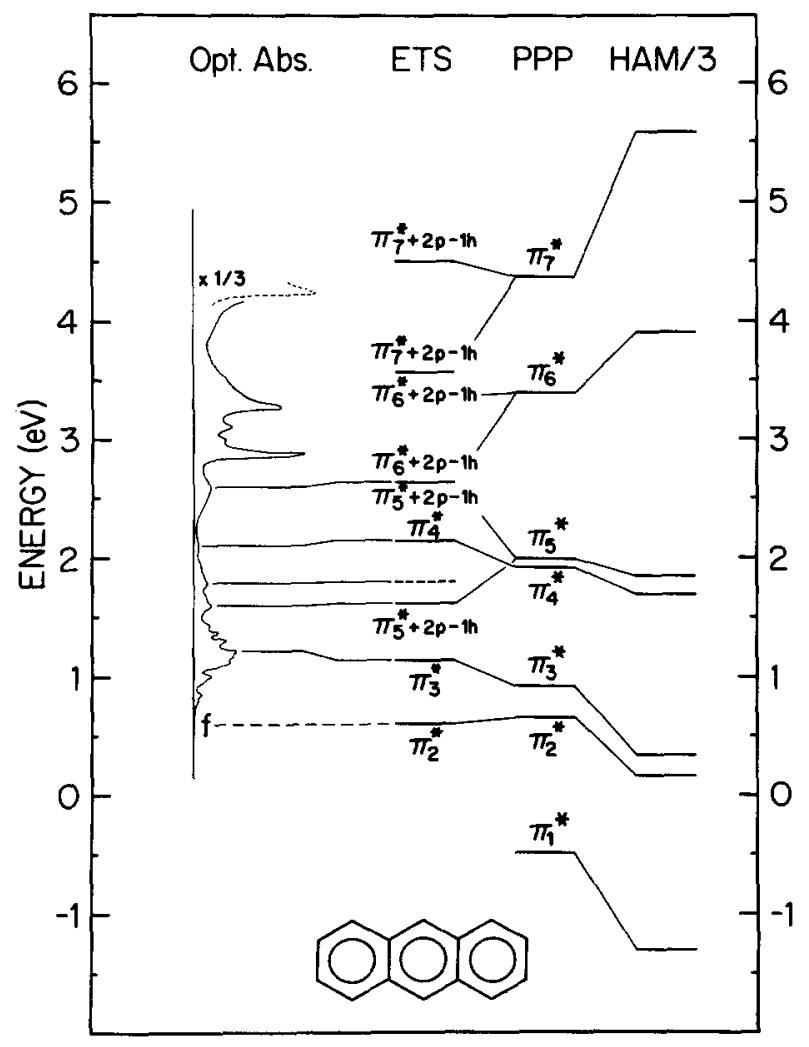

FIG. 7. A comparison of anthracene anion energies derived from ETS, optical absorption measurements on the anion in solution and PPP and HAM calculations. The energy scale for the optical absorption data has been shifted so that its zero lies at $-0.5 \mathrm{eV}$ in agreement with pairing theorem predictions. of structure observed in the optical absorption spectrum. The PPP calculations predict two nearly degenerate levels $\left(b_{3 u}\right.$ and $b_{1 g}$ ) centered around $1.95 \mathrm{eV}$. The $b_{1 g}$ orbital is of the correct symmetry to mix with the lowest $2 \mathrm{p}-1 \mathrm{~h}$ configuration, and Shida and Iwata ${ }^{46}$ predict the two resulting ${ }^{2} B_{1 g}$ anion states to lie near 1.8 and $2.6 \mathrm{eV}$. Accordingly we assign the $1.67 \mathrm{eV}$ and the higher lying $2.67 \mathrm{eV}$ resonance to the two ${ }^{2} B_{1 g}$ states and interpret the weak structure at $1.8 \mathrm{eV}$ to vibrational excitation. Evidence for vibrational structure is also seen in the absorption spectrum. We conclude that the $2.15 \mathrm{eV}$ feature is due to the ${ }^{2} B_{3 u}$ anion state which is expected to be largely single particle in nature. This assignment appears reasonable because the resonance lies within $0.2 \mathrm{eV}$ of the PPP value and because the corresponding feature in the absorption spectrum is very weak, consistent with a dipole forbidden transition.

The PPP procedure predicts two more anion states at $3.39 \mathrm{eV}\left(a_{1 u}\right)$ and $4.37 \mathrm{eV}\left(b_{1 g}\right)$. Although these lie only 0.2 $\mathrm{eV}$ below features in the ET spectrum, the $\mathrm{CI}$ calculations lead one to expect considerable mixing of each of these with $2 \mathrm{p}-1 \mathrm{~h}$ configurations. Considering further that both the 2.63 and $3.57 \mathrm{eV}$ resonance profiles are somewhat out of the ordinary, we are led to suggest that the ${ }^{2} A_{u}$ "pairs" fall near 2.6 and $3.6 \mathrm{eV}$ while the two members of the ${ }^{2} B_{1 \mathrm{~g}}$ "pair" lie near 3.6 and $4.5 \mathrm{eV}$. The weak feature at $6.05 \mathrm{eV}$ is likely due to a higher lying $2 \mathrm{p}-1 \mathrm{~h}$ state.

\section{Tetracene}

Figures 8 and 9 display the ET spectrum and anion energies of tetracene, respectively. The zero in the optical absorption is taken to lie at $-1.0 \mathrm{eV}$ corresponding to an EA of $+1.0 \mathrm{eV}$ deduced from the pairing theorem. The PPP value is less stable by $0.15 \mathrm{eV}$. Only the three lowest anion energies were available from the PPP calculations of Younkin et al. ${ }^{41}$

The first structure in the ET spectrum at $0.4 \mathrm{eV}$ is in good agreement with the PPP energies of the second and third $\pi^{*}$ orbitals. Since neither is expected to undergo appreciable configuration mixing, we attribute the lowest feature to the unresolved ${ }^{2} B_{3 u}$ and ${ }^{2} B_{1 g}$ excited anion states.

Evidence for $\mathrm{a}^{2} B_{3 u}$ state in this region is provided by the optical absorption measurements on the ground state anion which show a fairly intense peak at $0.5 \mathrm{eV}$ on the energy scale

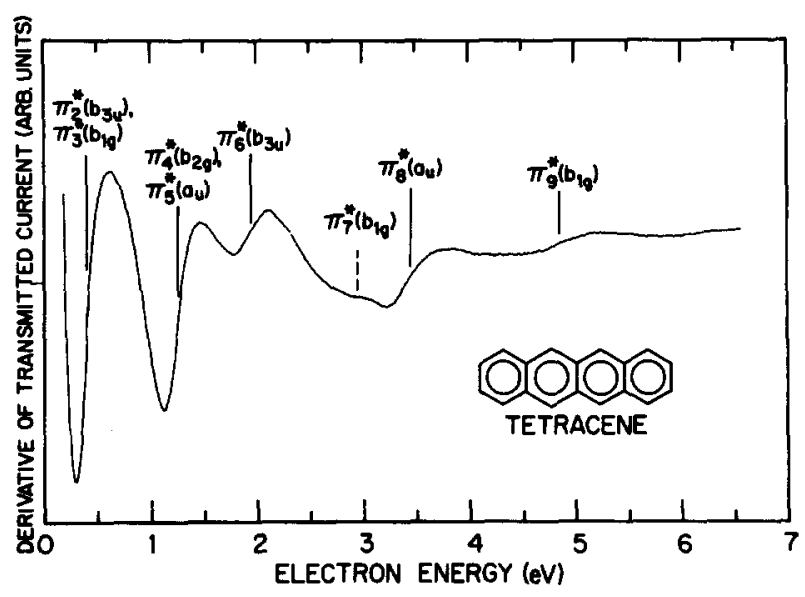

FIG. 8. Electron transmission spectrum of tetracene. 


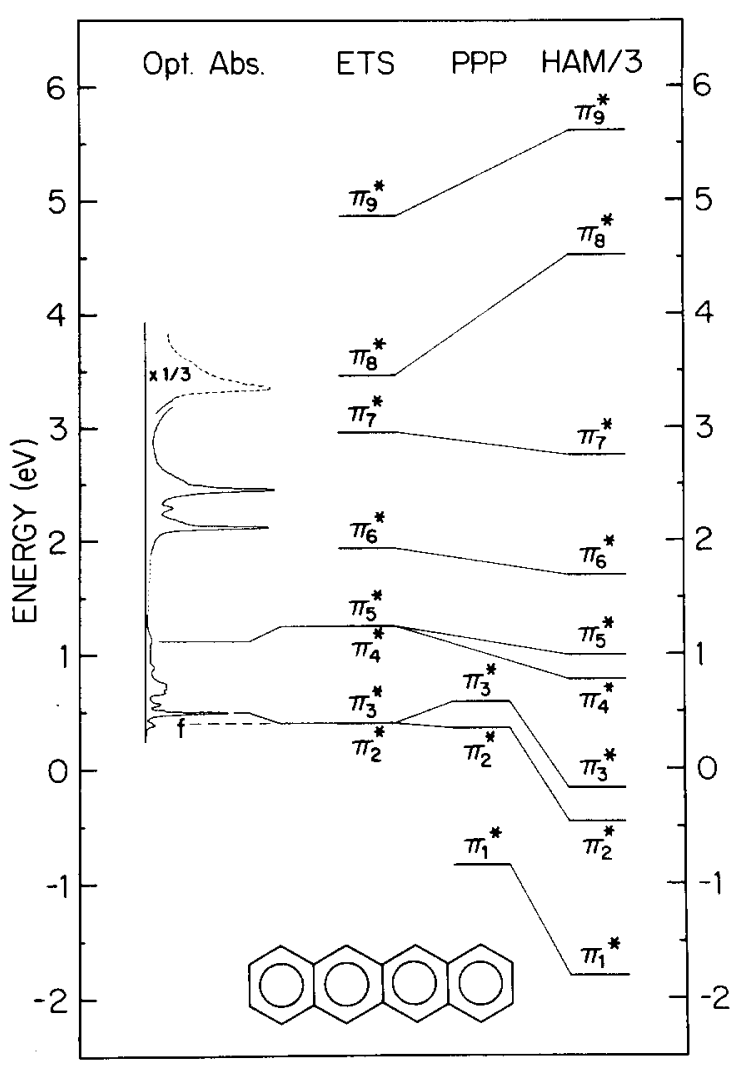

FIG. 9. A comparison of tetracene anion energies derived from ETS, optical absorption measurements on the anion in solution, PPP results (available only for the lowest three states), and HAM calculations. The energy scale for the optical absorption data has been shifted so that its zero lies at -1.0 $\mathrm{eV}$ in agreement with pairing theorem predictions.

used in Fig. 9. Moreover, the absorption spectrum, like that of naphthalene and anthracene, shows evidence of a weaker, vibronically induced transition in this region with structure starting at $0.4 \mathrm{eV}$, supporting our picture of an overlapping ${ }^{2} B_{1 g}$ anion state very close by.

At higher energies the optical absorption spectrum of the tetracene anion is considerably less useful for our purposes than those of naphthalene and anthracene, being dominated by transitions to the anion states which require "shake-up" to reach by electron attachment to the neutral. As noted before, the CI calculations greatly overestimate the splitting between pairs of anion states in the smaller acenes, and the results for tetracene appear even less reliable for our application.

The remaining resonances in Fig. 8 have been assigned on the basis of simple orbital considerations and designated by the appropriate 1 p orbital. We are guided primarily by the correspondence to the HAM/ 3 values shown in Fig. 9, taking into account their tendency to be increasingly stable below $1.7 \mathrm{eV}$ and increasingly unstable above. We can not rule out CI effects, but simply note that there are no obvious additional features in the ET spectrum which require such treatment. Because of the breadth of some of the resonances it is entirely possible, as in the case of the upper ${ }^{2} B_{1 \mathrm{~g}}$ resonance in naphthalene, that the related resonances in tetracene are spread over the adjacent features. These questions will ultimately have to be resolved by examining the decay channels of each of the resonances.

To summarize, the correlation diagram for the acenes is given in Fig. 10. The IP's are those of Clark et al ${ }^{50}$ and Munakata et al..$^{51}$ The correlation diagram is constructed using an "Aufbau" principle, that is, the orbitals of a given acene are constructed from those of the next smaller acene together with the appropriate orbitals of butadiene. The solid lines connect states of the same symmetry.

\section{B. Styrene, cis- and trans-stilbene}

\section{Styrene}

The ET spectra of styrene obtained under high and low electron rejection conditions are given in Fig. 11. The high rejection spectrum closely resembles that presented previously by us in an earlier communication. ${ }^{6(\mathrm{~b})}$ The lowest two resonances display considerable vibrational structure, and Figs. 12 and 13 show expanded views of these features.

As described earlier ${ }^{6(b)}$ and in more detail below, the lowest resonance is due to electron capture into the lowest unoccupied molecular orbital, which is composed of the bonding combination of the ethylenic $\pi^{*}$ orbital and one component of the benzene $e_{2 u} \pi^{*}$ orbital. Examination of the wave function of this molecular orbital suggests that vibrational modes involving a significant contribution from the ethylene $\mathrm{CC}\left(\mathrm{C}_{1}-\mathrm{C}_{2}\right)$ stretch and the C-ring $\left(\mathrm{C}_{2}-\mathrm{C}_{3}\right)$

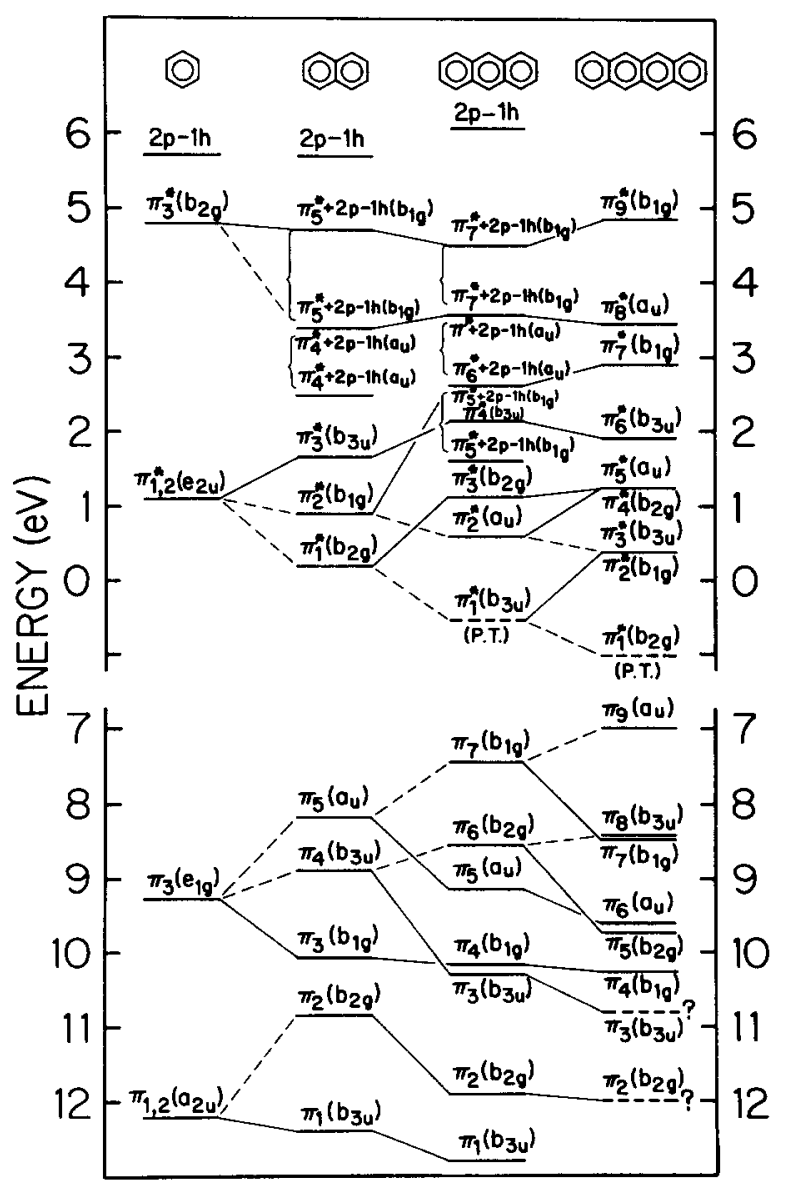

FIG. 10. Correlation diagram for the attachment energies and IP's (Refs. 50 and 51 ) of the linear acenes. States of the same symmetry are connected with solid lines. 


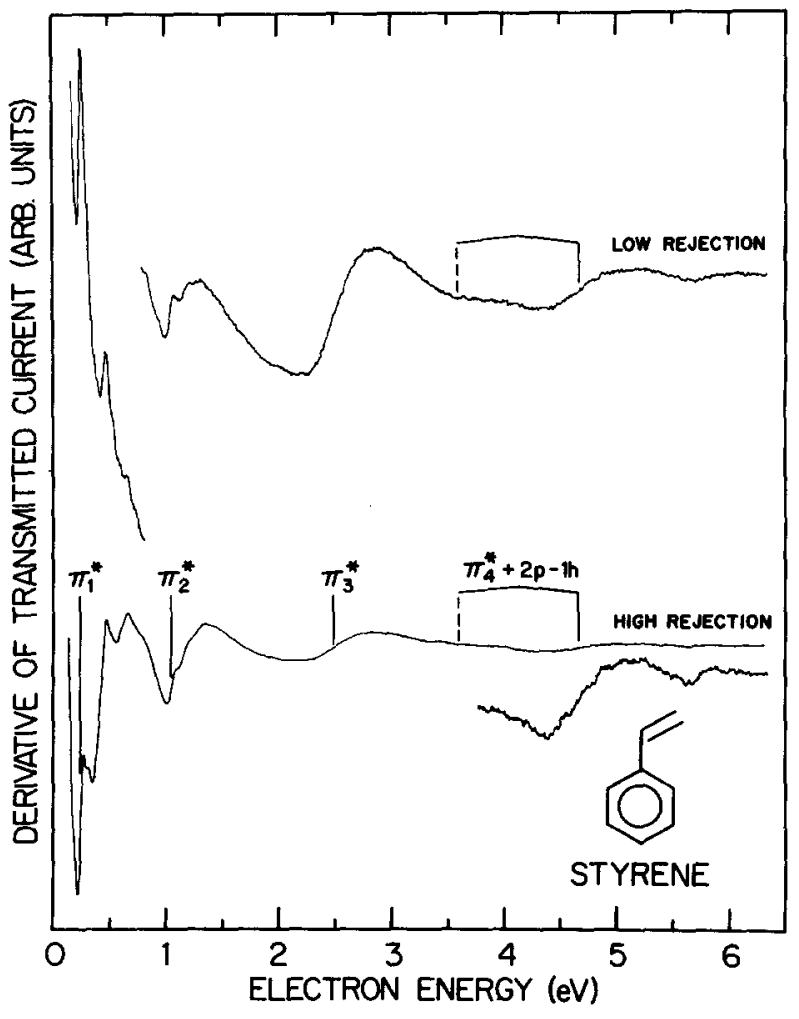

FIG. 11. Electron transmission spectra of styrene using high and low rejection of the scattered electrons.

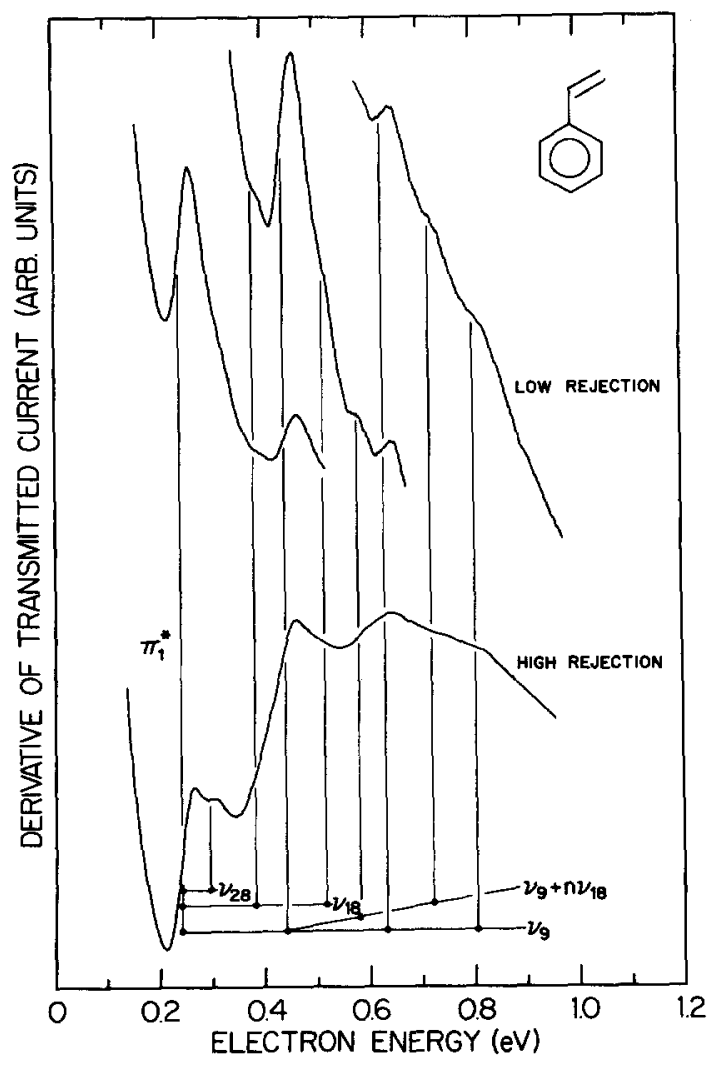

FIG. 12. Expanded views of the transmission spectra of styrene around the first resonance using high and low rejection of the scattered electrons.

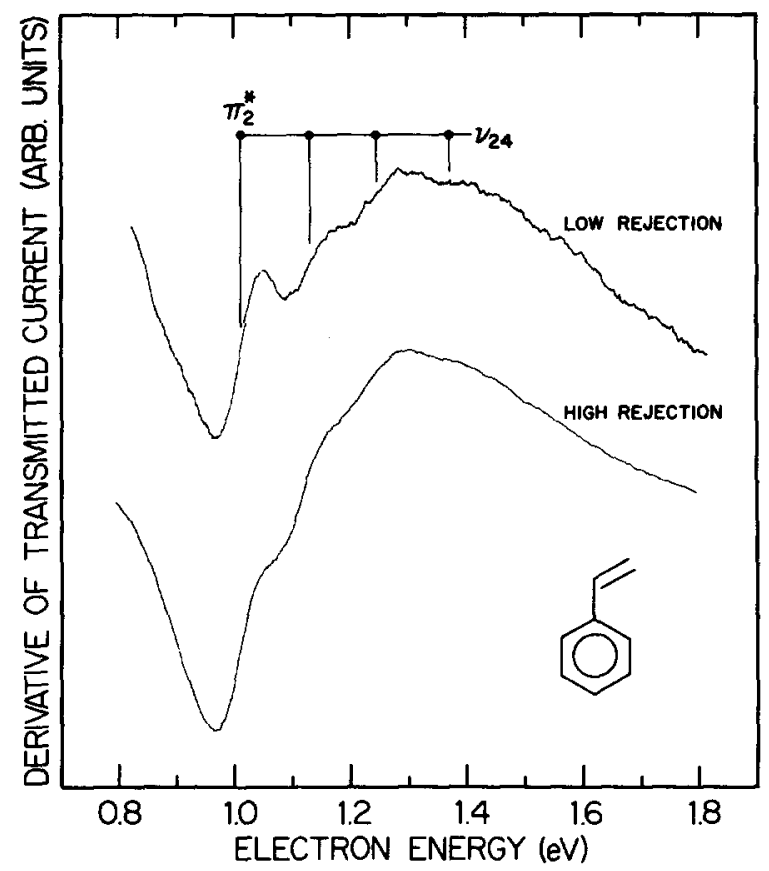

FIG. 13. Expanded views of the transmission spectra of styrene around the second resonance.

stretch are most likely to be stimulated upon electron capture.

The interpretation of the vibrational structure was greatly facilitated by the results of a recent theoretical study of Hemley $e t a l .^{52}$ These authors have decomposed the internal coordinate contributions in each of the normal modes of the ground state of styrene. Of the 29 planar normal modes, only 5 have appreciable motion, say $15 \%$ or more, along the $\mathrm{C}_{1}-\mathrm{C}_{2}$ or $\mathrm{C}_{2}-\mathrm{C}_{3}$ coordinates. This list could likely be reduced further by examining the detailed molecular motion in each mode, were this information available, and its compatibility with the impulse given the system by temporary occupation of $\pi_{1}^{*}$.

According to Hemley et al., the detailed make-up of these modes is as follows:

$v_{9}(202 \mathrm{meV})-33 \% \mathrm{C}_{1}-\mathrm{C}_{2}$ stretch; $32 \%$ ring $\mathrm{C}-\mathrm{C}$ stretch; $16 \%$ substituent bend; $14 \%$ ring bend; $5 \% \mathrm{C}_{2}-\mathrm{C}_{3}$ stretch.

$v_{11}(195 \mathrm{meV})-46 \%$ ring $\mathrm{C}-\mathrm{C}$ stretch; $22 \%$ ring bend; $18 \% \mathrm{C}_{1}-\mathrm{C}_{2}$ stretch; $11 \%$ substituent bend; $3 \% \mathrm{C}_{2}-\mathrm{C}_{3}$ stretch.

$v_{16}(162 \mathrm{meV})-61 \%$ substituent bend; $22 \% \mathrm{C}_{1}-\mathrm{C}_{2}$ stretch; 7\% $\mathrm{C}_{2}-\mathrm{C}_{1}$ stretch; 7\% ring bend; $3 \%$ ring $\mathrm{C}-\mathrm{C}$ stretch.

$v_{18}(149 \mathrm{meV})-31 \%$ substituent bend; $31 \%$ ring bend; $22 \% \mathrm{C}_{2}-\mathrm{C}_{3}$ stretch; $16 \%$ ring $\mathrm{C}-\mathrm{C}$ stretch.

$v_{28}(54 \mathrm{meV})-43 \%$ ring bend; $21 \%$ substituent bend; $19 \% \mathrm{C}_{2}-\mathrm{C}_{3}$ stretch; $13 \%$ ring $\mathrm{C}-\mathrm{C}$ stretch; $4 \% \mathrm{C}_{1}-\mathrm{C}_{2}$ stretch.

The high and low rejection data shown in Fig. 12 are remarkably complementary because of the differences in background, and certain of the features are more readily observed in one spectrum than the other. In additional data not 
shown, the rapidly falling background in the low rejection data was suppressed by a deliberate mistuning of the instrument, and the structure was made to appear on a more favorable slope. These data were also used in assigning the midpoints of the features shown in Fig. 12. The major progression in the ground state anion has spacings of 200 , 190 , and $175 \mathrm{meV}$, in excellent agreement with $v_{9}$ as observed in the neutral; while the low energy feature at $55 \mathrm{meV}$ or less is in agreement with $\boldsymbol{v}_{\mathbf{2 8}}$. The broadening of the major progression, particularly evident in the high rejection spectrum, is evidence for modes of intermediate energies, and indeed the upper curves show structure at approximately 140-150 meV, possibly due to $\boldsymbol{v}_{18}$. In Fig. 12 we have indicated the locations of these modes and certain of the combinations. The broadening at higher energies suggest that a number of weak nonresolved modes may well be present.

The structure in the second anion state shown in Fig. 13 is spaced at approximately $120 \mathrm{meV}$ and can reasonably be assigned to $v_{\mathbf{2 4}}$. Structure at approximately this spacing is also observed in the monosubstituted benzenes ${ }^{6(a)}$ in the anion states with similar charge distributions. Support for this assignment follows from the characteristics of the wave function of the second unoccupied $\pi^{*}$ orbital of styrene. Since this orbital is localized almost entirely on the ring, with nodes (in the Hückel or PPP approximations) at the carbon where the vinyl group is attached and at the para position, one expects excitation of a mode with little $\mathrm{C}_{1}-\mathrm{C}_{2}$ or $\mathrm{C}_{2}-\mathrm{C}_{3}$ motion. Examination of the modes tabulated by Hemley et al. reveals one strong candidate, $v_{24}(125 \mathrm{meV})$, described as $61 \%$ ring $\mathrm{C}-\mathrm{C}$ stretch; $36 \%$ ring bend; and $2 \%$ substituent bend. $\mathrm{A}$ number of other modes with slightly higher energies are also present with $1 \% \mathrm{C}_{1}-\mathrm{C}_{2}$ or $\mathrm{C}_{2}-\mathrm{C}_{3}$ activity.

With regard to the state assignments in the styrene anion, the ET spectrum of Fig. 11 provides clear-cut evidence for four anion states below an impact energy of $5 \mathrm{eV}$. The numbers and energies are consistent with expectations based on the interaction between the $\pi^{*}$ orbitals of the vinyl and phenyl moeities making up styrene. The assignments and energies are in good general agreement with those calculated from PPP and HAM/3. A correlation diagram constructed from the experimental EA's and IP's ${ }^{29,53-55}$ of ethylene, benzene, styrene, and stilbene, discussed below, is presented in Fig. 14.

The energies of the first three anion states of styrene are in excellent agreement with the prediction of PPP theory. Although the styrene molecule has $C_{1 h}$ symmetry, as a consequence of which all the $\pi^{*}$ orbitals have a" symmetry, ${ }^{56}$ it is convenient to view the molecule as having $C_{2 v}$ symmetry, in which case the first three $\pi^{*}$ orbitals in order of increasing energy are $b_{1}, a_{2}$, and $b_{1}$. The CI calculations of Shida et al. ${ }^{46}$ confirm the single particle nature of these three anion states. We note also that the energies of the first two transitions observed in the optical absorption spectrum ${ }^{57}$ of the anion in a rigid matrix agree within $0.2 \mathrm{eV}$ with the values deduced from the spacings between the energies of the anions of the gas phase molecule.

PPP theory predicts the fourth $\pi^{*}\left(b_{1}\right)$ orbital to be at $4.21 \mathrm{eV}$, whereas the ET spectrum has a strong feature at $4.67 \mathrm{eV}$ and a weak feature near $3.6 \mathrm{eV}$. The optical absorp-

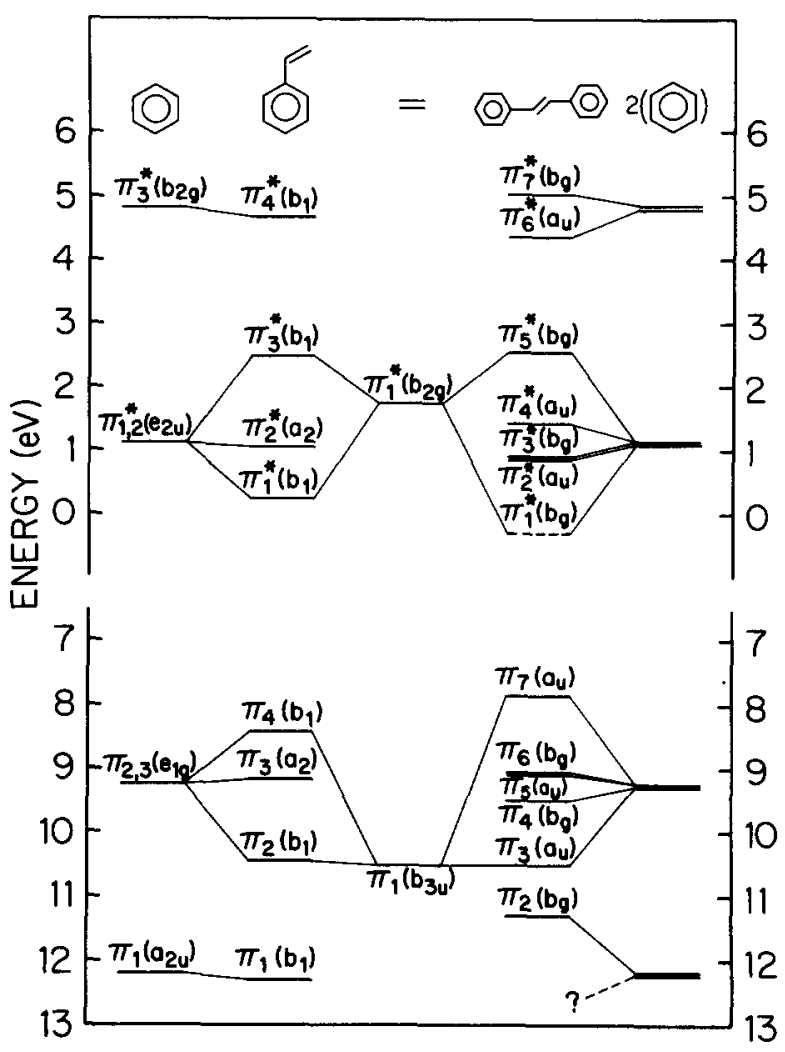

FIG. 14. Correlation diagram for the attachment energies and IP's (Refs. 29 and 53-55) of ethylene, benzene, styrene, and trans-stilbene.

tion studies ${ }^{57}$ show a broad, intense feature at $3.3 \mathrm{eV}$, the zero of energy being taken to correspond to the neutral molecule in the gas phase. Unfortunately the absorption spectrum was not reported above $4 \mathrm{eV}$. The CI calculations suggest that the $3.3 \mathrm{eV}$ feature in the absorption studies derives from two transitions involving configuration mixing between the $1 \mathrm{p} \pi_{4}^{*}$ configuration and $2 \mathrm{p}-1 \mathrm{~h}$ configurations. They also predict a state near $4.3 \mathrm{eV}$, derived from the same configurations. We therefore conclude that both the strong $4.67 \mathrm{eV}$ peak and the weak $3.6 \mathrm{eV}$ feature seen in ETS derive from states involving mixing of the $\pi_{4}^{*}$ orbital with $2 \mathrm{p}-1 \mathrm{~h}$ configurations. The $5.7 \mathrm{eV}$ feature in the ET spectrum is expected to be $2 \mathrm{p}-1 \mathrm{~h}$ in nature.

It should be noted that the $2 \mathrm{p}-1 \mathrm{~h}$ states manifest themselves in the ET spectrum of benzene above $5 \mathrm{eV}$. Given the changes in the first IP and first EA in going from benzene to styrene, one would expect the first $2 \mathrm{p}-1 \mathrm{~h}$ configuration to occur 1-1.5 eV lower in styrene. Hence, it is not at all surprising that the $\pi_{4}^{*}$ orbital mixes strongly with $2 \mathrm{p}-1 \mathrm{~h}$ configurations.

\section{Cls- and trans-st/lbene}

The electron transmission spectra of the two isomers of stilbene are compared in Fig. 15. As expected, the spectra for the two isomers are very similar, with the largest energy difference between corresponding features being less than $0.18 \mathrm{eV}$. Since the first anion states of benzene and styrene are at 1.12 and $0.24 \mathrm{eV}$, respectively, one would expect the lowest anion states of the stilbene to be stable and, hence, 


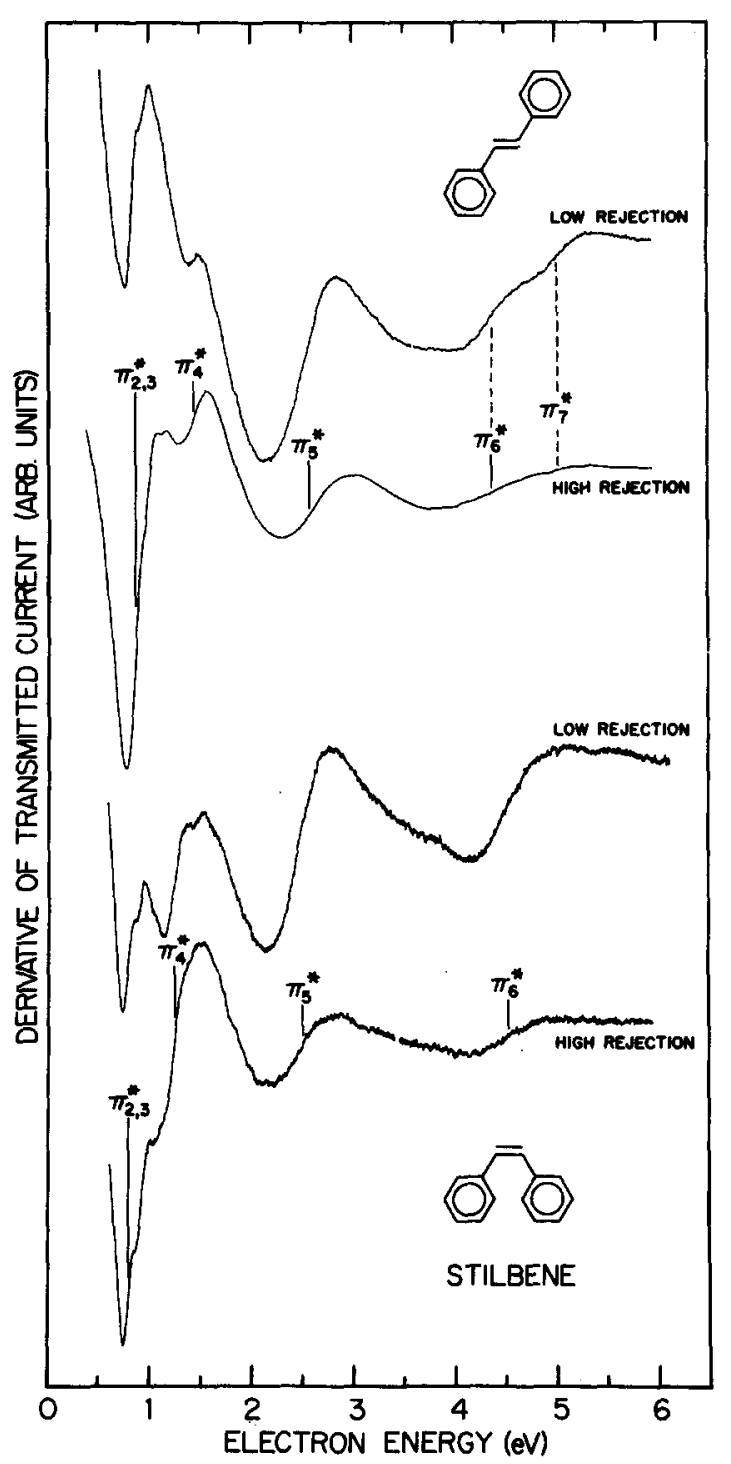

FIG. 15. Electron transmission spectra of cis- and trans-stilbene using both high and low rejection of scattered electrons.

inaccessible to direct observation by ETS. This is confirmed by the absence of structure at low energy in the ET spectrum and by the PPP and HAM/3 calculations which predict a stable anion.

The first features seen in the ET spectra are at 0.79 and $0.86 \mathrm{eV}$ for the cis and trans isomers, respectively. Each of these displays weak structure as well as a larger feature some 0.5 to $0.6 \mathrm{eV}$ higher in energy. Interpretation of these results is somewhat complicated by the fact that the PPP calculations predict three anion states between 0.96 and $1.18 \mathrm{eV}$.

The second and third $\pi^{*}$ orbitals, which in PPP theory have no charge distribution on the ethylenic bridging group, being formed from the two combinations of the $a_{2}$ components of the benzene $e_{2 u} \pi^{*}$ orbital, are accidentally degenerate in the PPP procedure as a result of the restriction to nearest neighbor interactions and the use of idealized geometries. The inclusion of non-nearest neighbor interactions would result in a small splitting between the second and third $\pi^{*}$ orbitals and would also introduce small differences between the energies of the orbitals of the two isomers.
Adoption of the correct geometries in the calculations, noting that both isomers are nonplanar, ${ }^{58}$ with torsion angles of $30^{\circ}$ and $43^{\circ}$ in the trans and cis species, respectively, will also act to remove the degeneracy and introduce additional differences between the spectra of the two isomers.

The lowest resonance in each isomer exhibits structure spaced at $120 \mathrm{meV}$ which could be due to vibrational structure or to the electronic splitting mentioned above. Since the structure in the stilbene spectrum between $0.8-1.0 \mathrm{eV}$ resembles, both in spacing and intensity, the vibrational structure observed in the second anion state of styrene, we favor the former interpretation, concluding that the second and third anion states are nearly degenerate (i.e., that they are split at most by a few hundredths of an $\mathrm{eV}$ ). We thus associate the 1.25 (cis) and $1.43 \mathrm{eV}$ (trans) features with the $\pi_{4}^{*}$ anion state predicted by the PPP calculations at $1.18 \mathrm{eV}$. Similarly, the resonance near $2.5 \mathrm{eV}$ in both isomers appears to be due to the $\pi_{5}^{*}$ anion state, which is predicted to be at $2.29 \mathrm{eV}$ in the PPP calculations. The higher lying $\pi^{*}$ resonances are completely overlapping in the range from 4.1 to $5.2 \mathrm{eV}$ except in the low rejection spectrum of trans-stilbene which shows evidence for two features. Along with $\pi_{6}^{*}$ and $\pi_{7}^{*}$, predicted to lie at 3.77 and $4.08 \mathrm{eV}$ in the PPP calculations, this region may well contain contributions from $2 \mathrm{p}-1 \mathrm{~h}$ anion states. CI calculations give several anion states in this energy region. ${ }^{47}$

The ET studies show that the EA's of the two isomers agree to within $0.18 \mathrm{eV}$. Given the $0.6 \mathrm{eV}$ difference between the second EA's of cis- and trans-hexatriene, ${ }^{7}$ one might have expected long-range through-space interactions to have caused greater differences between the energies of the anion states of the cis and trans isomers for which the extra electron occupies an orbital with appreciable charge density on the carbons brought into proximity in the cis structure. However, such interactions are less important in cis-stilbene because of the nonplanarity of the molecule and the smaller magnitude of the relevant MO coefficients.

The energy levels of the anion and cation states of cisand trans-stilbene are summarized along with those of ethylene, benzene, and styrene in Fig. 14. Our interpretation of the assignments of the anion states is in accord with that of the photoelectron spectrum. For example, Kobayashi et al. ${ }^{55}$ have concluded that the energies of the second and third cation states agree to within $0.2 \mathrm{eV}$ and that the fourth cation state is about $0.4 \mathrm{eV}$ above the second. To the extent that pairing is valid for cis- and trans-stilbene, this supports our assignment of the structure near $0.8 \mathrm{eV}$ to the second and third anion states and that at 1.25 (cis) or $1.43 \mathrm{eV}$ (trans) to the fourth anion state.

\section{CONCLUSIONS}

Electron transmission spectroscopy has been employed to characterize the vertical electron attachment processes in a series of alternant hydrocarbons. Pronounced vibrational structure is associated with the ground state anions of styrene and naphthalene as well as in the extensively studied benzene molecule. Although the ET spectrum of benzene displays structure due only to the ring breathing mode, that of these other two compounds shows structure due to a low 
frequency mode as well as the high frequency ( $\mathrm{C}-\mathrm{C}$ stretch) mode. Pairing considerations lead one to expect similar vibrational structure in the first PES band and the ground state anion. However, comparison of the ET and PE spectra show that the situation is more complicated. For example, the PE spectrum of benzene shows stronger excitation of the Jahn-Teller modes than of $v_{2}$, whereas only the latter is seen in the ET spectrum. Also, the first PE bands of styrene and naphthalene show little evidence of the low frequency modes which are found in the anion states.

The first excited anion states of styrene, naphthalene, and stilbene display weak vibrational structure. For naphthalene the structure in the excited state is exceptionally weak and due to a low frequency mode. In styrene and stilbene the structure, which is due to a high frequency mode, is somewhat more pronounced but weaker than that in benzene. Since the energies of these states are essentially the same as that of the benzene anion, the weakened visibility of the vibrational structure is likely due primarily to symmetry lowering, i.e., the admission of lower $l$ values in the resonant scattering. The second anion states in these molecules result from electron capture into orbitals which have charge distributions very similar to the $a_{2}$ component of the benzene $e_{2 u}$ $\pi^{*}$ orbital from which they are derived. The change in visibility is a measure of the sensitivity of the anion lifetimes to variations in local symmetry. For comparison, we note that in aniline and phenol the corresponding anions states do not display vibrational structure. ${ }^{6(a)}$ In these cases the anions lie several tenths of an $\mathrm{eV}$ above the ${ }^{2} E_{2 u}$ anion of benzene, and the energy destabilization combined with the symmetry lowering result in anion lifetimes short compared to the vibrational period. On the other hand, the ${ }^{2} A_{2}$ anion state of fluorobenzene $^{6(\mathrm{a})}$ is stabilized with respect to the ${ }^{2} E_{2 u}$ benzene anion and shows more pronounced vibrational structure than in styrene or stilbene but less than in benzene.

The energies of the low-lying anion states of all the alternant hydrocarbons studied here are in good agreement with the prediction of PPP calculations which neglect configuration mixing, the energies of the states deduced from absorption studies, as well as the values predicted from the pairing theorem, i.e., derived from the ionization potentials. This supports the characterization of these states as single particle states. The energies of the higher lying anion states do not agree as well with the PPP predictions. In addition, there are more features in the ET spectra at high energies than can be accounted for in a simple orbital picture. Two-particle-onehole configurations are responsible for the appearance of the "extra" features and account qualitatively, in many cases, for the deviations in energy from the predictions of PPP theory. However, we note that the energies of many of the higher lying anion states as determined from the ET measurements differ by several tenths of an $\mathrm{eV}$ from those predicted from pairing considerations or from PPP-CI calculations.

There are several factors which contribute to the failure of the pairing approximation and PPP-CI calculations for the higher lying states. The first of these is sigma-pi mixing brought about by configuration interaction, the effects of which will increase as the energy increases. Since the degree of sigma-pi mixing differs for the anion and cation states, this causes deviations from pairing. Secondly, PPP, PPP-CI, and HAM calculations as well as the pairing theorem all ignore the fact that the resonant anion states are mixed with the continuum. This mixing is responsible for the lifetimes of the resonances and results in their wave functions having a "continuum-like" component as well as a "bound state-like" component. This has several important ramifications. First, as the strength of coupling to the continuum increases, the anion lifetimes decrease and the continuum component of the wave function becomes larger, or, in other words, the anion wave function becomes less localized. This introduces shifts in the energies of the levels, with the shifts in general being larger as the lifetimes decrease. The coupling of the anion states to the continuum can affect the degree of mixing between configurations both due to the level shifts and the indirect coupling through the continuum. Given two configurations, one of which is strongly coupled to the continuum (short lifetime) and the other only weakly coupled to the continuum (long lifetime), the degree of mixing should be less than that which would result if the coupling to the continuum were weak for both.

In a previous paper we also raised the possibility that anion states with very short lifetimes $\left(<\sim 10^{-15} \mathrm{~s}\right)$ may not be fully electronically relaxed. In a time dependent picture, if the "residence time" for the scattered electron on the molecule is sufficiently short, the other electrons will not undergo the same degree of reorganization as if the residence time is long, and as a result the energy will be higher than expected based on theoretical methods neglecting the coupling to the continuum. While this undoubtedly plays a role for very short lived resonances, the present work seems to indicate that the qualitative trends in the higher lying anion states observed here are satisfactorily explained in terms of configuration mixing.

Comparison of the ET spectra with the absorption spectra of the ground state anions in solution reveals several important differences. First, several states which appear as pronounced resonances in the electron scattering measurements are absent or are very weak in the optical absorption studies because the transition from the anion ground state is dipole forbidden. On the other hand, some of the largest peaks in the absorption studies are due to transitions to dipole allowed two-particle-one-hole states which can be reached by one electron excitation from the ground state anion. The corresponding states are absent or appear only weakly in the ET spectrum, since in this experiment their formation requires a "two electron" process. The corresponding differences between the structures seen in the PE spectra and the absorption spectra of the cations have been noted by many researchers. ${ }^{48,49}$ The differences between the gas phase and condensed phase anion spectra are somewhat greater. In part this may be due to the consequences of the short lifetimes of the higher lying gas phase anions mentioned above. The lifetimes tend to be appreciably longer for the anions in the condensed phase. In fact, several anion states which are broad and featureless in the ET spectrum show sharp vibrational structure when studied in rigid matrices.

The availability of experimental data on a collection of alternant hydrocarbons allows one to evaluate the utility of 
semiempirical procedures such as the PPP and HAM methods. Considering only the simplest forms of these theories, i.e., those which ignore configurational mixing, the PPP predictions for the low lying EA's are in very good agreement with the experimental values. They fare less well at higher energies, for the reasons elaborated earlier. The HAM predictions, on the other hand, agree well with those of the PPP method and the ET results only for attachment energies near $1.7 \mathrm{eV}$. For lower energy anion states the HAM method yields results which are too low, while those for higher states lie considerably above the PPP predictions. Furthermore, the deviations of the HAM results from the PPP values increase as the energies depart from $1.7 \mathrm{eV}$. If the electron affinities as determined by the HAM procedure are scaled as follows: (EA/1.37) -0.45 (in $\mathrm{eV}$ ), the results are considerably closer to the PPP predictions. ${ }^{59}$ It should be noted that both of these methods account for the relaxation effects associated with electron attachment, the HAM calculations via the half-electron procedure and the PPP calculations by means of a constant shift of all energy levels.

\section{ACKNOWLEDGMENTS}

We gratefully acknowledge the support of the National Science Foundation (CHE-8317439 and CHE-7916816) during the course of this work. We wish to thank Dr. A. E. Howard, Dr. A. R. Johnston, Dr. A. Modelli, and Dr. N. S. Chiu for assistance with some of the spectra, Dr. P. Carsky and Dr. R. Zahradnik for sending us the results of their CI calculations, and Dr. R. Compton for sending us a copy of his PPP program.

'A general review of temporary negative ion formation in atoms and diatomic molecules is given by G. J. Schulz, Rev. Mod. Phys. 45, 378, 423 (1973).

${ }^{2} \mathrm{~A}$ brief review of the applications of electron transmission spectroscopy to hydrocarbon molecules is given by K. D. Jordan and P. D. Burrow, Acc. Chem. Res. 11, 341 (1978).

${ }^{3}$ (a) L. Sanche and G. J. Schulz, Phys. Rev. A 5, 1672 (1972); (b) 6, 69, 2500 (1972); (c) J. Chem. Phys. 58, 479 (1973); (d) I. Nenner and G. J. Schulz, ibid. 62, 1747 (1975).

${ }^{4}$ T. Koopmans, Physica 1, 104 (1934).

${ }^{5}$ P. D. Burrow and K. D. Jordan, Chem. Phys. Lett. 36, 594 (1975).

${ }^{\circ}$ (a) K. D. Jordan, J. A. Michejda, and P. D. Burrow, J. Am. Chem. Soc. 98, 7189 (1976); (b) P. D. Burrow, J. A. Michejda, and K. D. Jordan, ibid. 98,6392 (1976).

${ }^{7}$ P. D. Burrow and K. D. Jordan, J. Am. Chem. Soc. 104, 5247 (1982).

${ }^{8} \mathrm{~L}$. Salem, The Molecular Orbital Theory of Conjugated Systems (Benjamin, New York, 1966).

${ }^{9}$ C. A. Coulson and G. S. Rushbrooke, Proc. Cambridge Philos. Soc. 36, 193 (1940); A. D. McLachlen, Mol. Phys. 4, 49 (1961); J. Koutecky, J. Chem. Phys. 44, 3702 (1966); 47, 1501 (1967).

${ }^{10}$ H. C. Longuet-Higgins and J. A. Pople, Proc. Phys. Soc. A 68, 591 (1955).

${ }^{11}$ K. D. Jordan and P. D. Burrow, Chem. Phys. 45, 171 (1980).

${ }^{12}$ A. Stamatovic and G. J. Schulz, Rev. Sci. Instrum. 39, 1752 (1968); 41, 423 (1970).

${ }^{13}$ A. R. Johnston and P. D. Burrow, J. Electron. Spectrosc. Relat. Phenom. 25, 119 (1982).

${ }^{14}$ J. N. H. Brunt, G. C. King, and F. H. Read, J. Phys. B 10, 1289 (1977).
${ }^{15}$ P. D. Burrow, J. A. Michejda, and J. Comer, J. Phys. B 9, 3225 (1976). ${ }^{16}$ We assume here that the Franck-Condon principle holds for these attachment processes. We point out however that the approximations behind this principle may break down. This has been noted, for example, in the $N \rightarrow V\left(\pi \rightarrow \pi^{*}\right)$ transition of ethylene, S. D. Peyerimhoff and R. J. Buenker, Theor. Chim. Acta 27, 243 (1972).

${ }^{17}$ P. D. Burrow and L. Sanche, Phys. Rev. Lett. 28, 333 (1972); M. J. Boness and G. J. Schulz, Phys. Rev. A 9, 1969 (1974).

${ }^{18}$ C. E. Klots and R. N. Compton, J. Chem. Phys. 67, 1779 (1977)

${ }^{19}$ M. J. W. Boness, I. W. Larkin, J. B. Hasted, and L. Moore, Chem. Phys. Lett. 1, 292 (1967)

${ }^{20}$ K. D. Jordan, J. A. Michejda, and P. D. Burrow, J. Am. Chem. Soc. 98 , 1295, 7189 (1976).

${ }^{21}$ D. Mathur and J. B. Hasted, J. Phys. B 9, L31 (1976).

${ }^{22}$ J. R. Frazier, L. G. Christophorou, J. G. Carter, and H. C. Schweinler, J. Chem. Phys. 69, 3807 (1978).

${ }^{23}$ M. Allan, Helv. Chim. Acta 65, 2008 (1982).

${ }^{24}$ S. F. Wong and G. J. Schulz, Phys. Rev. Lett. 35, 1429 (1975).

${ }^{25}$ J. N. Bardsley and J. Mandl, Rep. Progr. Phys. 31, 471 (1968).

${ }^{26}$ L. G. Christophorou, D. L. McCorkle, and J. G. Carter, J. Chem. Phys. 60, 3779 (1974).

${ }^{27}$ G. J. Schulz, Phys. Rev. 112, 150 (1958).

${ }^{28} \mathrm{G}$. Herzberg, Infrared and Raman Spectra (Van Nostrand Reinhold, New York, 1945); G. Herzberg, Electronic Spectra of Polyatomic Molecules (Van Nostrand Reinhold, New York, 1966).

${ }^{29}$ L. Åsbrink, E. Lindholm, and O. Edquist, Chem. Phys. Lett. 5, 609 (1970).

${ }^{30}$ A. L. Hinde, D. Poppinger, and L. Radom, J. Am. Chem. Soc. 100, 4681 (1978).

${ }^{31}$ N. O. Lipari, C. B. Duke, and L. Pietronero, J. Chem. Phys. 65, 1165 (1976).

${ }^{32}$ J. E. Wertz and J. R. Bolton, Electron Spin Resonance: Elementary Theory and Practical Applications (McGraw-Hill, New York, 1972).

${ }^{33}$ R. Azria and G. J. Schulz, J. Chem. Phys. 62, 573 (1975). See also K. C. Smyth, J. A. Schiavone, and R. S. Freund, ibid. 61, 1782, 1789 (1974).

${ }^{34}$ R. N. Compton, R. H. Huebner, P. W. Reinhardt, and L. G. Christophorou, J. Chem. Phys. 48, 901 (1968).

${ }^{35}$ M. N. Pisanias, L. G. Christophorou, and J. G. Carter, Chem. Phys. Lett. 13, 433 (1972).

${ }^{36}$ D. Mathur and J. B. Hasted, Chem. Phys. Lett. 48, 50 (1977).

${ }^{37} \mathrm{M}$. Allan, Lecture Notes in Chemistry, edited by F. A. Gianturco and G. Stefani (Springer, Berlin 1984), Vol. 35, p. 14.

${ }^{38}$ P. A. Clark, F. Brogli, and E. Hielbronner, Helv. Chim. Acta 55, 1415 (1972).

${ }^{39}$ L. M. Sverdlov, M. A. Kovner, and E. P. Krainov, Vibrational Spectra of Polyatomic Molecules (Wiley, New York, 1974), p. 365.

${ }^{40}$ The leading $l$ values in partial wave expansions may be found in tables from F. H. Read, J. Phys. B 1, 893 (1968).

${ }^{41}$ J. M. Younkin, L. J. Smith, and R. N. Compton, Theor. Chim. Acta (Berl.) 41, 157 (1976); R. N. Compton, Y. Yoshioka, and K. D. Jordan, Theor. Chim. Acta 54, 529 (1980).

${ }^{42}$ L. Asbrink, C. Fridh, and D. Lindholm, Chem. Phys. Lett. 52, 72 (1977); E. Lindholm and L. Åsbrink, Molecular Orbitals and Their Energies Studied by the Semiempirical HAM Method, Lecture Notes in Chemistry, Vol. 38 (Springer, Berlin, 1985).

${ }^{43}$ L. Åsbrink, C. Fridh, and E. Lindholm, Z. Naturforsch. Teil A 33, 172 (1978).

${ }^{44}$ W. E. Wentworth, E. Chen, and J. E. Lovelock, J. Phys. Chem. 70, 445 (1966).

${ }^{45}$ L. G. Christophorou and E. L. Chaney, J. Chem. Phys. 52, 2165 (1970).

${ }^{46}$ T. Shida and S. Iwata, J. Am. Chem. Soc. 95, 3473 (1973).

${ }^{47}$ R. Zahradnik and P. Carsky, J. Phys. Chem. 74, 1240 (1970).

${ }^{48}$ T. Bally, S. Nitsche, K. Roth, and E. Haselbach, J. Am. Chem. S $\propto$. 106, 3927 (1984).

${ }^{49}$ J. Spanget-Larsen, Croatica Chem. Acta 57, 991 (1984).

${ }^{50}$ P. A. Clark, F. Brogli, and E. Heilbronner, Helv. Chim. Acta 55, 1415 (1972).

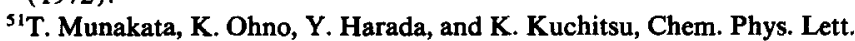
83, 243 (1981).

${ }^{52}$ R. J. Hemley, D. G. Leopold, V. Vaida, and M. Karplus, J. Chem. Phys. 82, 5379 (1985).

${ }^{53}$ M. Beez, G. Bieri, H. Bock, and E. Heilbronner, Helv. Chem. Acta 56, $1028(1973)$.

${ }^{54}$ M. J. S. Dewar and S. D. Worley, J. Chem. Phys. 50, 654 (1969). 
${ }^{55}$ T. Kobayashi, K. Yokota, and S. Nagakura, Bull. Chem. Soc. Jpn. 48, 412 (1975).

${ }^{56}$ L. A. Carreira and T. G. Towns, J. Chem. Phys. 63, 5283 (1975).

${ }^{57}$ T. Shida, S. Iwata, and M. Imamura, J. Phys. Chem. 78, 741 (1974).

${ }^{58}$ M. Traetteberg, E. B. Frantsen, F. C. Mijlhoff, and A. Hoekstra, J. Mol.
Struct. 26, 57 (1975); M. Traetteberg and E. B. Frantsen, ibid. 26, 69 (1975).

${ }^{39}$ The fitting parameters were derived from anion states in naphthalene. No attempt was made to optimize them over the set of molecules discussed here. 